\title{
UNRAMIFIED COHOMOLOGY OF QUADRICS, I
}

\author{
BRUNO KAHN, MARKUS ROST, AND R. SUJATHA
}

\begin{abstract}
Given a quadric $X$ over a field $F$ of characteristic $\neq 2$, we compute the kernel and cokernel of the natural map in degree 4 from the mod 2 Galois cohomology of $F$ to the unramified mod 2 cohomology of $F(X)$, when $\operatorname{dim} X>$ 10 and in several smaller-dimensional cases. Applications of these results to real quadrics and to the unramified Witt ring are given.
\end{abstract}

\section{Contents}

Introduction

Part I. Collected results on quadrics

1. Chow groups

Part II. $\quad \operatorname{Ker} \eta_{2}^{4}$ for higher-dimensional quadrics 14

3. Generalities on $\operatorname{Ker} \eta_{2}^{i} \quad 14$

4. 6-dimensional spinors $\quad 15$

5. $\operatorname{Ker} \eta_{2}^{4}$ and $H^{2}\left(X, \mathcal{K}_{3}\right) \quad 17$

6. Computations for $\operatorname{dim} X=3 \quad 19$

Part III. Coker $\eta_{2}^{i}$ for higher-dimensional quadrics $\quad 21$

7. A relationship between Ker $\eta_{2}^{i}$ and Coker $\eta_{2}^{i} \quad 21$

8. Constructing some maps 22

9. The case $i=2$ : Proof of theorem $4 \quad 28$

10. The case $i=3$ : Proof of theorem $5 \quad 28$

11. The case $i=4 \quad 31$

12. Proof of theorems 6 and $7 \quad 33$

Appendix A. Quadrics of dimension 0 and $1 \quad 35$

Appendix B. Application to real quadrics 36

Appendix C. The unramified Witt ring 37

References

\section{Introduction}

Let $F$ be a field, let $X$ be a smooth, proper, and irreducible variety over $F$ and let $n$ be prime to char $F$. Consider the restriction map

$$
H^{i}\left(F, \mu_{n}^{\otimes(i-1)}\right) \rightarrow H^{i}\left(F(X), \mu_{n}^{\otimes(i-1)}\right)
$$

in Galois cohomology.

The kernel of this map has been studied in various cases, for unirational and notably for homogeneous varieties. In the case of the Brauer group $(i=2)$ this goes

Date: August 23, 1997. 
back to Witt [56] for conics and Amitsur [2] for Severi-Brauer varieties. For $i=3$ the kernel has been determined by Arason [1] for quadrics. Other computations for $i=3$ and homogeneous varieties (Suslin [49], Peyre [39, 40], Merkurjev [29], Esnault et al. [14]) rely on the Merkurjev-Suslin theorem (see below). The MerkurjevSuslin/Rost theorem makes it possible to study also the case $i=4$ and $n=2$. Here certain quadrics have been considered by Jacob-Rost [16], Szyjewski [51], Rost and Merkurjev [27].

Unramified cohomology. The image of this map sits inside the unramified cohomology group

$H_{n r}^{i}\left(F(X) / F, \mu_{n}^{\otimes(i-1)}\right):=\operatorname{Ker}\left(\left(H^{i} F(X), \mu_{n}^{\otimes(i-1)}\right) \stackrel{\left(\partial_{x}\right)}{\longrightarrow} \bigoplus_{x \in X^{(1)}} H^{i-1}\left(F_{x}, \mu_{n}^{\otimes(i-2)}\right)\right)$.

Here $x$ runs through the codimension 1 points of $X$, and

$$
\partial_{x}: H^{i}\left(F(X), \mu_{n}^{\otimes(i-1)}\right) \rightarrow H^{i-1}\left(F_{x}, \mu_{n}^{\otimes(i-2)}\right)
$$

is the residue map associated to the discrete valuation ring $\mathcal{O}_{X, x}$.

We study the cokernel of the corresponding map

$$
\eta_{n}^{i}: H^{i}\left(F, \mu_{n}^{\otimes(i-1)}\right) \rightarrow H_{n r}^{i}\left(F(X) / F, \mu_{n}^{\otimes(i-1)}\right) .
$$

The group $H_{n r}^{i}\left(F(X) / F, \mu_{n}^{\otimes(i-1)}\right)$ is a birational invariant and does not change when $X$ is replaced by $X \times \mathbf{P}^{1}$. Moreover for the base field extension from $F$ to the rational function field $K=F(t)$ one has an exact sequence

$$
\begin{aligned}
0 \rightarrow H_{n r}^{i}\left(F(X) / F, \mu_{n}^{\otimes(i-1)}\right) \rightarrow H_{n r}^{i} & \left(K(X) / K, \mu_{n}^{\otimes(i-1)}\right) \rightarrow \\
& \rightarrow \bigoplus_{y \in \mathbf{A}_{(0)}^{1}} H_{n r}^{i-1}\left(F_{y}(X) / F_{y}, \mu_{n}^{\otimes(i-2)}\right) \rightarrow 0 .
\end{aligned}
$$

(cf. [7, §3.3, th. 4.1.1, 4.1.5], [42, (7.3),(12.10)].)

Saltman [43, 44], Bogomolov [3], Colliot-Thélène-Ojanguren [8], and Peyre [38], inter alia, have studied the unramified cohomology of certain unirational varieties over an algebraically closed $F$ (there are many more references available on this case, for which the reader can consult for instance $[7, \S 4]$ ). The unramified cohomology of real varieties has been studied in high cohomological degree by Colliot-ThélèneParimala [9] and Scheiderer [46]. For quadrics $X$ and $i \leq 3$, the cokernel of $\eta_{2}^{i}$ was studied by Colliot-Thélène-Sujatha [10] $(F=\mathbf{R})$, Peyre [39] ( $F$ arbitrary, $X$ a conic), Sujatha [48] ( $F$ arbitrary, $X$ defined by a neighbour of a 3 -fold Pfister form) and Kahn [17] ( $F$ arbitrary, $\operatorname{dim} X>2)$. It follows from [56, 1, 17] that $\eta_{2}^{2}$ $\left(\right.$ resp. $\left.\eta_{2}^{3}\right)$ is bijective for $\operatorname{dim} X>2($ resp. $\operatorname{dim} X>6)$ and has kernel and cokernel of order at most 2 for all $X$ (resp. for $\operatorname{dim} X>2$ ).

In this paper, we show that $\eta_{2}^{4}$ is bijective if $X$ is a quadric of dimension $>10$, and that $\left|\operatorname{Ker} \eta_{2}^{4}\right| \leq 2$, $\mid$ Coker $\eta_{2}^{4} \mid \leq 4$ for $\operatorname{dim} X>4$. To our knowledge, this is the first non-trivial instance where unramified cohomology of degree 4 is determined over an arbitrary field.

The Milnor conjecture. Before a more detailed discussion we recall the BlochKato conjecture, the Milnor conjecture, and the related conjecture about existence of cohomological invariants for the graded Witt ring. 
The Bloch-Kato conjecture predicts that if $n$ is invertible in $F$, then the norm residue homomorphism $h_{i}$ of degree $i$,

$$
h_{i}: K_{i}^{\mathrm{M}} F / n \rightarrow H^{i}\left(F, \mu_{n}^{\otimes i}\right)
$$

is an isomorphism for all $i \geq 0$. Here $K_{i}^{\mathrm{M}} F$ denotes the $i$-th Milnor $K$-group of $F$ [34]. If $n$ is a power of 2 , this is called the Milnor conjecture. The bijectivity of $h_{i}$ holds trivially for $i=0$ and by Kummer theory for $i=1$. The MerkurjevSuslin theorem states that $h_{2}$ is an isomorphism [31] and Merkurjev-Suslin/Rost [32] proved the Milnor conjecture for $i=3$. The second author announced a proof of the Milnor conjecture for $i=4$. Voevodsky [54] recently released a proof of the Milnor conjecture for all $i$. In the present paper we use only the Milnor conjecture for $i \leq 3$, except in appendix $\mathrm{C}$ for an application to the unramified Witt group which relies on the Milnor conjecture for $i=4$.

Let $W(F)$ denote the Witt ring of $F$ and let $\left(I^{i} F\right)_{i \geq 0}$ be the filtration on $W(F)$ given by the higher powers of the fundamental ideal $I(F)([26,45])$. For $a_{1}, a_{2}$, $\ldots, a_{i} \in F^{*}$ let $\left\langle\left\langle a_{1}, \ldots, a_{i}\right\rangle\right\rangle$ denote the $i$-fold Pfister form $\left\langle 1,-a_{1}\right\rangle \otimes \cdots \otimes\left\langle 1,-a_{i}\right\rangle$ (ibid.). A quadratic form $q$ is a neighbour of an $i$-fold Pfister form $\varphi$ if $\operatorname{dim} q>2^{i-1}$ and $q$ is similar to a subform of $\varphi$. For simplicity, write

$$
H^{i} F=H^{i}(F, \mathbf{Z} / 2) .
$$

We define a symbol to be an element of $H^{i} F$ of the form $\left(a_{1}, \ldots, a_{i}\right)$, where $a_{j} \in F^{*}$, $\left(a_{j}\right)$ is the class of $a_{j}$ in $H^{1} F$ by Kummer theory and $\left(a_{1}, \ldots, a_{i}\right):=\left(a_{1}\right) \cdot \ldots \cdot\left(a_{i}\right)$ is the cup-product. Let $P_{i}(F)$ denote the set of classes of $i$-fold Pfister forms in $W(F)$. There are maps

$$
e^{i}: P_{i}(F) \rightarrow H^{i} F
$$

which send the class of an $i$-fold Pfister form $\left\langle\left\langle a_{1}, \ldots, a_{i}\right\rangle\right\rangle$ to the corresponding cohomological symbol $\left(a_{1}, \ldots, a_{i}\right)$. It is conjectured that these maps extend to well-defined isomorphisms

$$
\bar{e}^{i}: I^{i} F / I^{i+1} F \rightarrow H^{i} F
$$

for all $i \geq 0$. For $i \leq 2$, it is easy to see that these maps are well-defined, being the rank mod 2, signed discriminant, and the Clifford invariant, respectively, for $i=0$, 1, 2. Arason [1] proved that $\bar{e}^{3}$ is well-defined and Jacob-Rost/Szyjewski [16, 51] proved that $\bar{e}^{4}$ is well-defined. Granting the existence of $\bar{e}^{i}$, the Milnor conjecture in degree $i$ implies the bijectivity of $\bar{e}^{i}$. Voevodsky has recently announced a proof of the existence and bijectivity of the homomorphisms $\bar{e}^{i}$ for all $i$, jointly with Orlov and Vishik [35].

We now describe our results. Let $q$ be a (regular) quadratic form over a field $F$ with char $F \neq 2$ and let $X$ be the associated projective quadric. Unless mentioned otherwise, we assume $\operatorname{dim} q \geq 3$.

Note that if $q$ is isotropic, then the extension $F(X) / F$ is purely transcendental and therefore $\eta_{2}^{i}$ is bijective for all $i$ (cf. proposition 2.5).

Kernel of $\eta_{2}^{i}$. Here the major result is

Theorem 1 (cf. theorem 6.3). Let $i \leq 4$. If $\operatorname{dim} q=2^{i-2}+1$, then every element in $\operatorname{Ker} \eta_{2}^{i}$ is a symbol. 
For $i=3$ and, in case of a Pfister neighbour, for $i=4$, this theorem follows easily from the computations of Ker $\eta_{2}^{i}$ by Arason [1], Jacob-Rost [16], and Szyjewski [51, 6.1].

The proof of theorem 1 for $i=4$ in general is an extension of the discussions in $[16,27]$ to arbitrary 5 -dimensional quadratic forms. The method used there provides a very general description of $\operatorname{Ker} \eta_{2}^{4}$ for quadratic forms of dimension $\geq 5$ (see $[27])$ as follows:

$$
\operatorname{Ker} \eta_{2}^{4}=\operatorname{Ker}\left(H^{2}\left(X, \mathcal{K}_{3}\right) \rightarrow H^{2}\left(\bar{X}, \mathcal{K}_{3}\right)\right) .
$$

For an Albert form with corresponding biquaternion algebra $A$ (see below) this yields an isomorphism $\mathrm{SK}_{1}(\mathrm{~A})=\operatorname{Ker} \eta_{2}^{4}$ (ibid.).

For a five dimensional form $q$, let $\widehat{q}=q \perp\langle-d\rangle, d=\operatorname{det}(q)$ be the attached Albert form. In this case $(* *)$ yields a surjective homomorphism

$$
\mathrm{O}(\widehat{q}) /[\mathrm{O}(\widehat{q}), \mathrm{O}(\widehat{q})] \mathrm{O}(q) \rightarrow \operatorname{Ker} \eta_{2}^{4}
$$

Theorem 1 follows from a careful computation of this map.

Using this theorem and some quadratic form theory, we show in section 3:

Corollary 2. Let $i \leq 4$. Then

(1) A symbol $\left(a_{1}, \ldots, a_{i}\right) \neq 0$ lies in $\operatorname{Ker} \eta_{2}^{i}$ if and only if $q$ is similar to a subform of $\left\langle\left\langle a_{1}, \ldots, a_{i}\right\rangle\right\rangle$.

(2) $\operatorname{Ker} \eta_{2}^{i}$ consists of symbols for $\operatorname{dim} q>2^{i-2}$.

(3) $\left|\operatorname{Ker} \eta_{2}^{i}\right| \leq 2$ for $\operatorname{dim} q>2^{i-1}$.

(4) $\operatorname{Ker} \eta_{2}^{i}=0$ for $\operatorname{dim} q>2^{i}$.

Note that the bound $\operatorname{dim} q>2^{i-2}$ in (2) is optimal: if $q$ is an $(i-2)$-fold Pfister form $\left\langle\left\langle a_{1}, \ldots, a_{i-2}\right\rangle\right\rangle$, then $\operatorname{Ker} \eta_{2}^{i}$ contains $H^{2} F \cdot\left(a_{1}, \ldots, a_{i-2}\right)$, which obviously contains non-symbolic elements in general.

One may expect that theorem 1 also holds for higher degree $i$. Then corollary 2 would hold accordingly (using the existence and bijectivity of the $\bar{e}^{i}$ ).

Cokernel of $\eta_{2}^{i}$. Results on Coker $\eta_{2}^{i}$ are best understood in terms of Coker $\eta^{i}$, where $\eta^{i}$ is the restriction map

$$
\eta^{i}: H^{i}(F, \mathbf{Q} / \mathbf{Z}(i-1)) \rightarrow H_{n r}^{i}(K / F, \mathbf{Q} / \mathbf{Z}(i-1)) .
$$

Here $\mathbf{Q} / \mathbf{Z}(i-1):=\lim _{\longrightarrow} \mu_{m}^{\otimes(i-1)}$, where $m$ runs through all integers prime to char $F$. A consequence of Milnor's conjecture in degree $i-1$ is that $H^{i} F \rightarrow$ $H^{i}(F, \mathbf{Q} / \mathbf{Z}(i-1))$ is injective; hence $\operatorname{Ker} \eta_{2}^{i}=\operatorname{Ker} \eta^{i}$ since the latter group is killed by 2 ( $c f$. lemma 7.2 a)). To relate Coker $\eta_{2}^{i}$ and Coker $\eta^{i}$, define

$$
\left(\operatorname{Ker} \eta_{2}^{i}\right)_{0}=\left\{\alpha \in \operatorname{Ker} \eta_{2}^{i} \mid(-1) \cdot \alpha=0 \in H^{i+1} F\right\} .
$$

We prove in section 7 :

Proposition 3 ( $c f$. proposition 7.4). For any quadric $X$ and $i \leq 4$, there is an exact sequence

$$
0 \rightarrow\left(\operatorname{Ker} \eta_{2}^{i}\right)_{0} \rightarrow \text { Coker } \eta_{2}^{i} \rightarrow \text { Coker } \eta^{i} .
$$

This way, information on $\operatorname{Ker} \eta_{2}^{i}$ and Coker $\eta^{i}$ immediately yields information on Coker $\eta_{2}^{i}$.

To describe our picture about Coker $\eta^{i}$ we formulate:

\section{Conjecture.}


(1) Coker $\eta^{i}=0$ if $q$ is Pfister neighbour.

(2) Coker $\eta^{i}=0$ for $\operatorname{dim} q \leq 5$.

(3) Coker $\eta^{i}$ is finite for $\operatorname{dim} q>6 \cdot 2^{i-4}$.

(4) Coker $\eta^{i}=0$ for $\operatorname{dim} q>6 \cdot 2^{i-3}$.

In appendix $\mathrm{A}$ we show Coker $\eta^{i}=0$ for $\operatorname{dim} q=3$ and-after an appropriate modification of the target group - also for $\operatorname{dim} q=2$. Here the arguments rely on the Milnor conjecture in degree $i-2$ (resp. $i-1)$.

For $i \leq 2$ one has ( $c f$. section 9$)$ :

Theorem 4. Coker $\eta^{i}=0$ for $i \leq 2$.

For $i=3$ there appears a peak in the story at $\operatorname{dim} q=6$. For the reader's convenience, we recall the various types of 6 -dimensional quadratic forms. Let $d_{ \pm} q$ be the signed discriminant and let $C(q)$ be the Clifford algebra of $q[26]$. The pure subform $\left\langle\left\langle a_{1}, \ldots, a_{i}\right\rangle\right\rangle^{\prime}$ of the Pfister form $\left\langle\left\langle a_{1}, \ldots, a_{i}\right\rangle\right\rangle$ is defined by

$$
\left\langle\left\langle a_{1}, \ldots, a_{i}\right\rangle\right\rangle=\langle 1\rangle \perp\left\langle\left\langle a_{1}, \ldots, a_{i}\right\rangle\right\rangle^{\prime} .
$$

There are four types of anisotropic quadratic forms $q$ of dimension 6 :

- $d=d_{ \pm} q \neq 1, q_{F(\sqrt{d})}$ is anisotropic. For convenience, we shall call such forms virtual Albert forms.

- $d_{ \pm} q=1$, i.e., $q \in I^{2} F$. Such forms are called (anisotropic) Albert forms. An Albert form is similar to a form of type

$$
\langle\langle a, b\rangle\rangle^{\prime} \perp-\langle\langle e, f\rangle\rangle^{\prime} \quad a, b, e, f \in F^{*} .
$$

To an Albert form there is attached the biquaternion algebra $A=(a, b) \otimes$ $(e, f)$ which is the central simple algebra with $C(q) \simeq M_{2}(A)$. The form $q$ is isotropic if and only if $A$ is not a skewfield.

- $d=d_{ \pm} q \neq 1, q_{F(\sqrt{d})}$ is hyperbolic. These forms are the 6 -dimensional Pfister neighbours (cf. [24, p. 10]).

- $d=d_{ \pm} q \neq 1, q_{F(\sqrt{d})}$ is isotropic without being hyperbolic. Such forms are of type $a \tau \perp b\langle 1,-d\rangle$, where $a, b \in F^{*}$ and $\tau$ is a 2-fold Pfister form.

Now for $i=3$ our result is ( $c f$. section 10):

Theorem 5. Coker $\eta^{3}=0$, except if $q$ is an anisotropic Albert form, for which Coker $\eta^{3} \simeq \mathbf{Z} / 2$.

This result completes those of [17].

The main results about Coker $\eta^{4}$ are:

Theorem 6 ( $c f$. section 12).

(1) For $\operatorname{dim} q>6$ there is an exact sequence

$0 \rightarrow$ Coker $\eta^{4} \rightarrow\left(C H^{3} X\right)_{\text {torsion }} \rightarrow\left(C H^{1} X \otimes H^{3}(F, \mathbf{Q} / \mathbf{Z}(2))\right) \oplus H^{5}(F, \mathbf{Q} / \mathbf{Z}(3))$.

Here $C H^{p} X$ denotes the $p$-th Chow group of $X$.

(2) $\mid$ Coker $\eta^{4} \mid \leq 2$ if $\operatorname{dim} q \neq 4,5,6$.

(3) Coker $\eta^{4}=0$ if

- $q$ is a Pfister neighbour

- $\operatorname{dim} q=8$ and $d_{ \pm} q=1$

- $\operatorname{dim} q>12$. 
In the proof of theorem 6 we use Karpenko's results on $C H^{3}$ of a quadric. The main computational tool is, as in [17], to mix the use of the Hochschild-Serre and Bloch-Ogus [4] spectral sequences, here with divisible coefficients. We use in particular the fact that these two spectral sequences are modules over the Galois cohomology of $F$. Divisible coefficients have been used also in $[23,6,39,40]$. It is clear that these methods can also be applied to other homogeneous varieties.

Theorem 7. If $q$ is an anisotropic Albert form or a virtual Albert form over F, then Coker $\eta^{4}$ is infinite for $q_{F(t)}$.

This theorem is an easy consequence of theorem 5 and the exact sequence $(*)$.

From corollary 2, proposition 3 , and theorem 6 one easily deduces:

\section{Corollary 8.}

(1) Coker $\eta_{2}^{2}=0$, unless $q$ is a neighbour of a 2 -fold Pfister form $\langle\langle a, b\rangle\rangle$. In this case, it is 0 or $\mathbf{Z} / 2$ according as $(-1, a, b) \in H^{3} F$ is nonzero or not.

(2) If $q$ is an anisotropic Albert form, $\left(\operatorname{Ker} \eta_{2}^{3}\right)_{0}=0$ and Coker $\eta_{2}^{3} \simeq \mathbf{Z} / 2$. In all other cases, Coker $\eta_{2}^{3} \simeq\left(\operatorname{Ker} \eta_{2}^{3}\right)_{0}$. In particular:

a) If $q$ is a neighbour of a 3 -fold Pfister form $\langle\langle a, b, c\rangle\rangle$, then Coker $\eta_{2}^{3}=0$ or $\mathbf{Z} / 2$ according as $(-1, a, b, c) \in H^{4} F$ is nonzero or not.

b) If $q$ is not an Albert form and does not embed into a 3-fold Pfister form (up to similarity), then Coker $\eta_{2}^{3}=0$.

(3) a) If $\operatorname{dim} q>12$ and $q$ is not a neighbour of a 4-fold Pfister form, then Coker $\eta_{2}^{4}=0$.

b) If $q$ is a neighbour of a 4-fold Pfister form $\langle\langle a, b, c, d\rangle\rangle$, then Coker $\eta_{2}^{4}=0$ or $\mathbf{Z} / 2$ according as $(-1, a, b, c, d) \in H^{5} F$ is nonzero or not.

c) For $\operatorname{dim} q>8$, $\mid$ Coker $\eta_{2}^{4} \mid \leq 4$.

In the case $F=\mathbf{R}$, these results immediately imply that Coker $\eta_{2}^{i}=0$ for all quadrics when $i \leq 4$. In appendix $\mathrm{B}$, we also get partial results on the $\mathcal{H}$ cohomology of such quadrics.

The unramified Witt ring. As in [17, corollary], we wish to apply the above results to the unramified Witt ring. Denote by $W_{n r}(K / F)$ the unramified Witt ring of a finitely generated extension $K$ of $F\left(c f\right.$. [8]) and, for $i \geq 0$, by $I_{n r}^{i}(K / F)$ the intersection $I^{i} K \cap W_{n r}(K / F)$. Let

$$
\chi^{i}: I^{i} F / I^{i+1} F \rightarrow I_{n r}^{i}(K / F) / I_{n r}^{i+1}(K / F)
$$

be the natural map analogous to $\eta_{2}^{i}$ and $\eta^{i}$. The homomorphism $\bar{e}^{i}$ induces a homomorphism

$$
I_{n r}^{i} K / I_{n r}^{i+1} K \rightarrow H_{n r}^{i} K
$$

and hence a homomorphism

$$
\text { Coker } \chi^{i} \rightarrow \text { Coker } \eta_{2}^{i}
$$

Using the Milnor conjecture in degree $i=4$, we find:

Theorem 9. Let $i \leq 4$. For any quadric $X$, the composition

$$
\text { Coker } \chi^{i} \rightarrow \text { Coker } \eta_{2}^{i} \rightarrow \text { Coker } \eta^{i}
$$

is injective.

From corollary 2, theorem 6 , and theorem 9 we deduce: 


\section{Corollary 10.}

(1) The map $W(F) / I^{3} F \rightarrow W_{n r}(F(X) / F) / I_{n r}^{3}(F(X) / F)$ is surjective. It is bijective for $\operatorname{dim} q>4$.

(2) The map $W(F) / I^{4} F \rightarrow W_{n r}(F(X) / F) / I_{n r}^{4}(F(X) / F)$ is surjective, except if $q$ is an anisotropic Albert form. In this case, its cokernel is $\simeq \mathbf{Z} / 2$ (by [17, remark 3, p. 249]). It is bijective for $\operatorname{dim} q>8$.

(3) The map $W(F) / I^{5} F \rightarrow W_{n r}(F(X) / F) / I_{n r}^{5}(F(X) / F)$ is surjective in cases

- $q$ is a Pfister neighbour

- $\operatorname{dim} q=8$ and $d_{ \pm} q=1$

- $\operatorname{dim} q>12$.

It is bijective for $\operatorname{dim} q>16$.

Remark. Voevodsky's work yields also the computation of Ker $\eta_{2}^{i}$ for $i$-fold Pfister forms. A consequence of [54] and [35] is the validity of proposition 3 and of theorem 9 for all $i$.

However these announcements do not imply a generalization of theorem 1 to higher degree or a proof of the conjecture in an obvious way.

In a sequel to this paper, we plan to complete the present results by determining $\operatorname{Ker} \eta^{4}$ and Coker $\eta^{4}$ in the cases pending here, and by refining results on real quadrics. In particular, we shall show that $\operatorname{Ker} \eta_{2}^{4}$ is always generated by its symbols. For this, we shall use a method different from that of this paper, based on the following result:

Theorem ([19]). Let $X$ be a quadric and let $C$ be a conic such that there exist a morphism $C \rightarrow X$. Let $D$ be the quaternion algebra associated to $C$. Then there is a canonical isomorphism

$$
\operatorname{Ker} \eta_{2}^{4} \stackrel{\sim}{\rightarrow} \frac{H^{0}\left(X, K_{2}^{D}\right)}{K_{2} D}
$$

Here $H^{0}\left(X, K_{2}^{D}\right)$ is the intersection of the kernels of the residue maps

$$
K_{2}\left(D \otimes_{F} F(X)\right) \rightarrow K_{1}\left(D \otimes_{F} F(x)\right),
$$

where $x$ runs through the points of codimension 1 in $X$.

This paper is organized as follows. In part I we recall some known results on Chow groups, in particular of Karpenko, and of $\mathcal{K}$ - and $\mathcal{H}$-cohomology of quadrics, supplying proofs when they don't appear in the literature. Part II is devoted to the proof of theorem 1 and corollary 2, and part III to the proof of proposition 3, theorem 5, and theorem 6. In appendix A we consider Coker $\eta^{i}$ for quadrics of dimension 0 and 1 . In appendix $\mathrm{B}$ we consider the case $F=\mathbf{R}$. In appendix $\mathrm{C}$ we prove theorem 9 .

Notation and conventions. For any scheme $X, H^{i} X$ denotes the $i$-th étale cohomology group of $X$ with coefficients $\mathbf{Z} / 2$. We use the abbreviation $H^{i}(X, j)$ for $H_{\text {ét }}^{i}(X, \mathbf{Q} / \mathbf{Z}(j))$ and similarly $H^{p}\left(X, \mathcal{H}^{q}(j)\right)$ for the $E_{2}$-term of the coniveau spectral sequence with divisible coefficients. If $X$ is smooth over a field $F$, we denote by $C H^{i} X$ its $i$-th Chow group, the group of cycles of codimension $i$ over $X$ modulo rational equivalence. We denote by $c l_{X}^{i}\left(\right.$ resp. $\left.c l_{X}^{i}{ }_{X}\right)$ the cycle map with $\mathbf{Z} / 2$ coefficients $C H^{i} X / 2 \rightarrow H^{2 i} X$ (resp. with divisible coefficients $C H^{i} X \otimes \mathbf{Q} / \mathbf{Z} \rightarrow$ $\left.H^{2 i}(X, \mathbf{Q} / \mathbf{Z}(i))\right)$. We set $\bar{X}=X \times_{F} F_{s}$, where $F_{s}$ is a separable closure of $F$. The 
word "quadric" means smooth, projective and absolutely irreducible quadric. If $X$ is an even-dimensional quadric over $F$ (assumed of characteristic $\neq 2$ ), we denote by $d(X) \in F^{*} / F^{* 2}$ the (signed) discriminant $d_{ \pm} q$ of any quadratic form $q$ defining $X$. If $\varphi: A \rightarrow B$ is a homomorphism between two abelian groups, we sometimes allow ourselves to write $B / A$ or $\frac{B}{A}$ for Coker $\varphi$, even if $\varphi$ is not injective.

Acknowledgements. The first author wishes to thank Andrei Suslin for a conversation which catalyzed the use of divisible coefficients in this paper, and gratefully acknowledges the hospitality of the Tata Institute of Fundamental Research, where this work was completed. The third author acknowledges the partial support of a NATO grant.

\section{Part I. Collected results on quadrics}

In this part, we state general results on Chow groups, $\mathcal{H}$ - and $\mathcal{K}$-cohomology of quadrics, most of which are "classical". The reader is advised to skip it and to use it only for reference purposes. We merely give references when published proofs are available, and provide proofs otherwise.

We say that a quadric $X$ is anisotropic (resp. isotropic, split) if a quadratic form defining $X$ is anisotropic (resp. is isotropic, has maximal index).

\section{Chow groups}

The following proposition is mainly a reformulation of results of N. Karpenko [20].

Proposition 1.1. Let $X$ be a quadric over a field $F$ of characteristic $\neq 2$. Let $h$ denote the class of a hyperplane section in $C H^{1} \mathrm{X}$.

a) For any $i \geq 0$, the natural map $C H^{i} X \rightarrow C H^{i} \bar{X}$ has kernel the torsion subgroup of $C H^{i} X$ (which is 2-primary).

b) For $i<\operatorname{dim} X / 2, C H^{i} X /$ torsion is generated by $h^{i} ; \mathrm{CH}^{i} \mathrm{X} \rightarrow \mathrm{CH}^{i} \bar{X}$ is surjective.

c) For $i>\operatorname{dim} X / 2, C H^{i} X /$ torsion is generated by $h^{i}$ if $i \leq \operatorname{dim} X-n$ and by the class of a rational linear subspace of codimension $i$ if $i>\operatorname{dim} X-n$. Here $n$ is the Witt index of a quadratic form defining $X$. $\operatorname{Coker}\left(C H^{i} X \rightarrow C H^{i} \bar{X}\right) \simeq$ $\mathbf{Z} / 2$ if $i \leq \operatorname{dim} X-n$ and $C H^{i} X \rightarrow C H^{i} \bar{X}$ is surjective if $i>\operatorname{dim} X-n$.

d) For $i=\operatorname{dim} X / 2$ and $d(X)=1$ one has

$$
C H^{i} X / \text { torsion } \simeq \mathbf{Z} \oplus \mathbf{Z},
$$

with basis $\left(h^{i}, 2^{t} l\right)$, where $t \geq 0$ and $l$ is the class of some maximal linear subspace of $\bar{X}$. Moreover $\operatorname{Coker}\left(\mathrm{CH}^{i} \mathrm{X} \rightarrow \mathrm{CH}^{i} \bar{X}\right) \simeq \mathbf{Z} / 2^{t}$. We have $t=1$ if $i=1$ and $X$ is anisotropic, and $t=2$ if $i=2$ and $X$ is anisotropic.

e) For $i=\operatorname{dim} X / 2$ and $d(X) \neq 1$ one has

$$
C H^{i} X / \text { torsion } \simeq \mathbf{Z}
$$

generated by $h^{i}$. The map $C H^{i} X \rightarrow\left(C H^{i} \bar{X}\right)^{G_{F}}$ is surjective, where $G_{F}$ is the absolute Galois group of $F$.

Proof. a), b), and c) follow easily from [20, (2.1), (2.2), and (2.7)]; d) and e) follow from the same references plus $[20,(2.4)$ and (5.5)]. 
Corollary 1.2. Let $X$ be a quadric over $F, D$ a divisible group and $i \geq 0$. If $i \neq \operatorname{dim} X / 2$ or $i=\operatorname{dim} X / 2$ and $d(X)=1$, there is an exact sequence

$$
0 \rightarrow \operatorname{Tor}\left(D, \operatorname{Coker}\left(C H^{i} X \rightarrow C H^{i} \bar{X}\right)\right) \rightarrow D \otimes C H^{i} X \rightarrow D \otimes C H^{i} \bar{X} \rightarrow 0 .
$$

This is clear, since $C H^{i} \bar{X}$ is torsion-free and the map $C H^{i} X \rightarrow C H^{i} \bar{X}$ has torsion kernel and cokernel.

Corollary 1.3. Let $X$ be a quadric of dimension $2 i$ over $F$ such that $d=d(X) \neq 1$, $E=F(\sqrt{d})$ and $\Gamma=\operatorname{Gal}(E / F)$. Then the $\Gamma$-module $C H^{i} X_{E} /$ torsion is isomorphic to $\operatorname{Ind}_{1}^{\Gamma} \mathbf{Z}$ if $X_{E}$ is split, and to $\mathbf{Z} \oplus \mathbf{Z}(\varepsilon)$ otherwise, where $\mathbf{Z}(\varepsilon)$ is the $\Gamma$-module of support $\mathbf{Z}$ with the nontrivial action of $\Gamma$. On the other hand, the $G_{F}$-module $C H^{i} \bar{X}$ is always isomorphic to $\operatorname{Ind}_{G_{E}}^{G_{F}} \mathbf{Z}$. The maps

$$
C H^{i} X / \text { torsion } \rightarrow\left(C H^{i} \bar{X}\right)^{G_{F}}
$$

and

$$
C H^{i} X \otimes \mathbf{Q} / \mathbf{Z} \rightarrow H^{0}\left(F, C H^{i} \bar{X} \otimes \mathbf{Q} / \mathbf{Z}\right)
$$

are isomorphisms.

Proof. By prop. $1.1 \mathrm{~d}), C H^{i} X_{E} /$ torsion is a free $\mathbf{Z}$-module with basis $\left(h^{i}, 2^{t} l\right)$ for some $t \geq 0$. It is clear that $t=0$ if and only if $l$ is rational over $E$, i.e., iff $X_{E}$ is split. In this case, another basis of $C H^{i} X_{E}$ / torsion is $\left(l, l^{\prime}\right)$ where $l^{\prime}=h^{i}-l$ is the class of another linear section, and this basis is permuted by $\Gamma$, hence the first claim. If $t>0$, another basis of $C H^{i} X_{E} /$ torsion is given by $\left(h^{i}, 2^{t-1} h^{i}-2^{t} l\right)$, and $2^{t-1} h^{i}-2^{t} l$ is a -1 -eigenvector for $\Gamma$. This proves the second claim. The isomorphism $C H^{i} X /$ torsion $\stackrel{\sim}{\longrightarrow}\left(C H^{i} \bar{X}\right)^{G_{F}}$ has been seen in prop. 1.1 a) and e). To get the other, we consider the short exact sequence of $G_{F}$-modules

$$
0 \rightarrow C H^{i} \bar{X} \rightarrow C H^{i} \bar{X} \otimes \mathbf{Q} \rightarrow C H^{i} \bar{X} \otimes \mathbf{Q} / \mathbf{Z} \rightarrow 0
$$

obtained by tensoring the exact sequence $0 \rightarrow \mathbf{Z} \rightarrow \mathbf{Q} \rightarrow \mathbf{Q} / \mathbf{Z} \rightarrow 0$ by the flat Z-module $C H^{i} \bar{X}$. Since $C H^{i} \bar{X} \simeq \operatorname{Ind}_{G_{E}}^{G_{F}} \mathbf{Z}$, we have $H^{1}\left(F, C H^{i} \bar{X}\right)=0$ by Shapiro lemma, hence we get an isomorphism

$$
C H^{i} X \otimes \mathbf{Q} / \mathbf{Z} \stackrel{\sim}{\longrightarrow}\left(C H^{i} \bar{X}\right)^{G_{F}} \otimes \mathbf{Q} / \mathbf{Z} \stackrel{\sim}{\longrightarrow} H^{0}\left(F, C H^{i} \bar{X} \otimes \mathbf{Q} / \mathbf{Z}\right) .
$$

Corollary 1.4. Let $X$ be an anisotropic quadric over $F, K=F(X)$ and $i_{1}(X)$ the Witt index of $q_{K}$, where $q$ is a quadratic form defining $X$.

a) For $i<\operatorname{dim} X / 2, C H^{i} X /$ torsion $\rightarrow C H^{i} X_{K} /$ torsion is bijective.

b) For $i>\operatorname{dim} X / 2, C H^{i} X /$ torsion $\rightarrow C H^{i} X_{K} /$ torsion is bijective if $i \leq$ $\operatorname{dim} X-i_{1}(X)$ and $\operatorname{Coker}\left(C H^{i} X /\right.$ torsion $\rightarrow C H^{i} X_{K} /$ torsion $) \simeq \mathbf{Z} / 2$ if $i>\operatorname{dim} X-i_{1}(X)$.

c) For $i=\operatorname{dim} X / 2, C H^{i} X /$ torsion $\rightarrow C H^{i} X_{K} /$ torsion is bijective if $d(X) \neq 1$. If $d(X)=1$, Coker $\left(C H^{i} X /\right.$ torsion $\rightarrow C H^{i} X_{K} /$ torsion $)$ is cyclic of order $2^{s}$ for some $s \leq t$, where $t$ is as in prop. $1.1 \mathrm{~d})$. For $i=1$ or 2 we have $s=1$.

d) For $i=\operatorname{dim} X / 2$ and $d=d(X) \neq 1$, let $E=F(\sqrt{d})$. Then

$$
C H^{i} X_{E} / \text { torsion } \rightarrow C H^{i} X_{K E} / \text { torsion }
$$

is bijective if $X_{E}$ is isotropic and has cokernel as in c) otherwise. 
Proof. Everything directly follows from proposition 1.1, except for the last sentence of c) and for d). Assume $\operatorname{dim} X=2$. Then $X$ is defined by an anisotropic quaternion form, (= a 2-fold Pfister form). hence $i_{1}(X)=2$, which implies $s=1$ in this case by proposition $1.1 \mathrm{~d}$ ). Assume $\operatorname{dim} X=4$. Then $X$ is defined by an anisotropic Albert form $\varphi$, hence $i_{1}(X)=1$. Let $Y$ be the quadric over $K$ given by the anisotropic part of $\varphi_{K}(\operatorname{dim} Y=2)$. By [20, (2.2)] (cf. lemma 2.1 below), there is an isomorphism $C H^{1} Y \stackrel{\sim}{\longrightarrow} C H^{2} X_{K}$. This isomorphism is natural under change of base field, as can be seen from the proof of lemma 2.1. In particular, the diagram

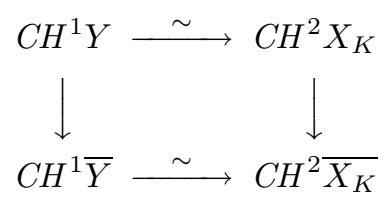

commutes, which shows that $\operatorname{Coker}\left(\mathrm{CH}^{2} \mathrm{X}_{K} \rightarrow C H^{2} \overline{X_{K}}\right) \simeq \mathbf{Z} / 2$. Moreover, since $\operatorname{Coker}\left(\mathrm{CH}^{2} \mathrm{X} \rightarrow \mathrm{CH}^{2} \bar{X}\right) \simeq \mathbf{Z} / 4$ and $\mathrm{CH}^{2} \bar{X} \stackrel{\sim}{\longrightarrow} \mathrm{CH}^{2} \overline{X_{K}}$, this indeed shows that $s=1$. Finally, d) is obvious since, if $X_{E}$ is isotropic, the extension $K E / E$ is purely transcendental (compare [20, proof of (3.12)]).

Proposition 1.5. Let $X$ be a quadric over $F$, where $F$ is separably closed (of characteristic $\neq 2)$. Then $H^{j} X=0$ for $j$ odd and the cycle maps

$$
c l_{X}^{i}: C H^{i} X / n \rightarrow H^{2 i}\left(X, \mu_{n}^{\otimes i}\right)
$$

are isomorphisms for any $n$ prime to $\operatorname{char} F$ and $i \geq 0$.

Proof. This follows from proposition 2.2 a) and the computation of $H_{\mathrm{ett}}^{*}\left(X, \mathbf{Z}_{l}\right)$ [11, th. 3.3].

Proposition 1.6. Let $X$ be a quadric over $F$.

a) (classical) $C H^{1} X$ is torsion-free.

b) $([20,(6.1)]) \quad\left(C H^{2} X\right)_{\text {torsion }} \simeq \mathbf{Z} / 2$ if $X$ is defined by a neighbour of an anisotropic 3-fold Pfister form, and is 0 otherwise.

c) $([20,(2.6)],[21],[22]) \quad\left(C H^{3} X\right)_{\text {torsion }} \simeq 0$ or $\mathbf{Z} / 2$. If $\operatorname{dim} X=3$ or $\operatorname{dim} X>$ $10,\left(C H^{3} X\right)_{\text {torsion }}=0$.

\section{2. $\mathcal{K}$ - and $\mathcal{H}$-cohomology}

We denote by $H^{i}\left(X, \mathcal{K}_{j}\right)$ and $H^{i}\left(X, \mathcal{H}^{j}\left(\mu_{m}^{\otimes k}\right)\right)$ the cohomology groups of the localization complexes with terms

$$
\bigoplus_{x \in X^{(i)}} K_{j-i} F(x) \quad \text { resp. } \quad \bigoplus_{x \in X^{(i)}} H^{j-i}\left(F(x), \mu_{m}^{\otimes(k-i)}\right) .
$$

These cohomology groups are defined for any equidimensional, not necessarily smooth variety. For the long exact excision sequences and for homotopy invariance we refer to [42]. The interpretation for smooth $X$ as Zariski cohomology groups is not needed here.

Lemma 2.1. Let $X$ be an isotropic quadric of dimension $n \geq 2$ over a field $F$ of characteristic $\neq 2$; write $q \simeq q^{\prime} \perp x_{n} x_{n+1}$ for a quadratic form defining $X$ and 
$Z \subseteq X$ for the codimension 2 subquadric with equation $q^{\prime}=0$. Then, for $0<i<n$, $j \geq i$ and $k \in \mathbf{Z}$, there are isomorphisms:

$$
\begin{aligned}
K_{j} F & \stackrel{\sim}{\longrightarrow} H^{0}\left(X, \mathcal{K}_{j}\right), \\
H^{i-1}\left(Z, \mathcal{K}_{j-1}\right) & \stackrel{\sim}{\longrightarrow} H^{i}\left(X, \mathcal{K}_{j}\right), \\
K_{j-n} F & \stackrel{\sim}{\longrightarrow} H^{n}\left(X, \mathcal{K}_{j}\right), \\
H^{j}\left(F, \mu_{m}^{\otimes k}\right) & \stackrel{\sim}{\longrightarrow} H^{0}\left(X, \mathcal{H}^{j}\left(\mu_{m}^{\otimes k}\right)\right), \\
H^{i-1}\left(Z, \mathcal{H}^{j-1}\left(\mu_{m}^{\otimes(k-1)}\right)\right) & \stackrel{\sim}{\longrightarrow} H^{i}\left(X, \mathcal{H}^{j}\left(\mu_{m}^{\otimes k}\right)\right), \\
H^{j-n}\left(F, \mu_{m}^{\otimes(k-n)}\right) & \stackrel{\sim}{\longrightarrow} H^{n}\left(X, \mathcal{H}^{j}\left(\mu_{m}^{\otimes k}\right)\right) .
\end{aligned}
$$

These isomorphisms commute with change of base field, transfer and cup-product by $K_{*} F\left(\operatorname{resp.} H^{*}\left(F, \mu_{m}^{\otimes l}\right)\right)$.

Remark. If $n=2$, the "subquadric" $Z$ is $\operatorname{Spec} E$, with $E=F[t] / t^{2}-d$, where $d=d(X)$ is the (signed) discriminant of the 2-dimensional form $q^{\prime} . E$ is a quadratic extension of $F$ if $d \neq 1$ and $F \times F$ otherwise.

Proof. (compare [16, lemma 3.3], [51, prop. 3.2.1] for $\operatorname{dim} X=3$, and [20, (2.2)] for $i=j$ ). We do it only for $\mathcal{K}$-cohomology, the case of $\mathcal{H}$-cohomology being identical. Let $Y$ be the hyperplane section $x_{n+1}=0$ and $P=(0: \cdots: 0: 1: 0)$. We have excision exact sequences:

$$
\begin{gathered}
\cdots \rightarrow H^{i-1}\left(Y, \mathcal{K}_{j-1}\right) \rightarrow H^{i}\left(X, \mathcal{K}_{j}\right) \rightarrow H^{i}\left(X \backslash Y, \mathcal{K}_{j}\right) \rightarrow H^{i}\left(Y, \mathcal{K}_{j-1}\right) \rightarrow \cdots \\
\cdots \rightarrow H^{i-n}\left(P, \mathcal{K}_{j-n}\right) \rightarrow H^{i-1}\left(Y, \mathcal{K}_{j-1}\right) \rightarrow H^{i-1}\left(Y \backslash P, \mathcal{K}_{j-1}\right) \rightarrow \\
\rightarrow H^{i-n+1}\left(P, \mathcal{K}_{j-n}\right) \rightarrow \cdots
\end{gathered}
$$

On the other hand, $X \backslash Y$ is isomorphic to the affine space and $P$ makes the projection $Y \backslash P \rightarrow Z$ a vector bundle with affine line fibres. Homotopy invariance of $\mathcal{K}$-cohomology yields new exact sequences and isomorphisms:

$$
\begin{gathered}
H^{i-1}\left(Y, \mathcal{K}_{j-1}\right) \stackrel{\sim}{\rightarrow} H^{i}\left(X, \mathcal{K}_{j}\right) \quad \text { if } i>1, \\
0 \rightarrow H^{0}\left(X, \mathcal{K}_{j}\right) \rightarrow K_{j} F \rightarrow H^{0}\left(Y, K_{j-1}\right) \rightarrow H^{1}\left(X, \mathcal{K}_{j}\right) \rightarrow 0
\end{gathered}
$$

and

$$
\begin{gathered}
H^{i-1}\left(Z, \mathcal{K}_{j-1}\right) \stackrel{\sim}{\longrightarrow} H^{i-1}\left(Y \backslash P, \mathcal{K}_{j-1}\right) \quad \text { if } i<n-2, \\
0 \rightarrow H^{n-2}\left(Y, \mathcal{K}_{j-1}\right) \rightarrow H^{n-2}\left(Z, \mathcal{K}_{j-1}\right) \rightarrow K_{j-n} F \rightarrow H^{n-1}\left(Y, \mathcal{K}_{j-1}\right) \rightarrow 0
\end{gathered}
$$

The composition $K_{j} F \rightarrow H^{0}\left(X, \mathcal{K}_{j}\right) \rightarrow K_{j} F$ is the identity, so that the exact sequence (2.2) splits as two isomorphisms. Similarly, for $n>0, K_{j-n} F \rightarrow$ $H^{n-1}\left(Y, \mathcal{K}_{j-1}\right) \stackrel{\sim}{\longrightarrow} H^{n}\left(X, \mathcal{K}_{j}\right)$ is induced by the inclusion of the closed point $P$ in $X$ and composition with the norm $H^{n}\left(X, \mathcal{K}_{j}\right) \stackrel{N}{\longrightarrow} K_{j-n} F$ is the identity, so the exact sequence (2.4) also splits as two isomorphisms. Putting all this together, we get the isomorphisms of lemma 2.1. The naturality statements are clear from the construction of these isomorphisms.

Proposition 2.2. Let $X$ be a split quadric over a field $F$ of characteristic $\neq 2$, and $h \in C H^{1} X$ the class of a hyperplane section. 
a) $C H^{i} X \simeq \mathbf{Z}$ for $i \neq \operatorname{dim} X / 2$, and $C H^{i} X \simeq \mathbf{Z} \oplus \mathbf{Z}$ if $i=\operatorname{dim} X / 2 ; h^{i}$ is a generator of $C H^{i} X$ for $i<\operatorname{dim} X / 2$ and twice a generator for $i>\operatorname{dim} X / 2$. For $i=\operatorname{dim} X / 2$, a basis of $C H^{i} X$ is given by the classes $l$, $l^{\prime}$ of two maximal linear subspaces of $X$ such that $h^{i}=l+l^{\prime}$.

b) The natural maps

$$
K_{j-i} F \otimes C H^{i} X \rightarrow H^{i}\left(X, \mathcal{K}_{j}\right)
$$

given by the Brown-Gersten-Quillen spectral sequence and cup-product are isomorphisms.

c) The natural maps

$$
H^{j-i}\left(F, \mu_{m}^{\otimes k}\right) \otimes C H^{i} X \rightarrow H^{i}\left(X, \mathcal{H}^{j}\left(\mu_{m}^{\otimes(i+k)}\right)\right)
$$

given by the coniveau spectral sequence and cup-product are isomorphisms for all $m \geq 1$.

Proof. a) follows from proposition 1.1. Let us prove b) (as above, the proof of c) is identical and omitted). (See [29, prop. 1 and prop. 3] for another approach.) The quadric $X$ is isomorphic to

$$
X_{n}: \begin{cases}x_{0} x_{1}+\cdots+x_{n} x_{n+1}=0 & \text { if } n \text { is even } \\ x_{0}^{2}+x_{1} x_{2}+\cdots+x_{n} x_{n+1}=0 & \text { if } n \text { is odd }\end{cases}
$$

where $n=\operatorname{dim} X$. As a consequence of lemma 2.1, the diagram

$$
\begin{array}{ccc}
H^{i-1}\left(X_{n-2}, \mathcal{K}_{j-1}\right) & \sim H^{i}\left(X_{n}, \mathcal{K}_{j}\right) & \\
\cup \uparrow & \cup \uparrow & \text { for } 0<i<n \\
K_{j-i} F \otimes C H^{i-1} X_{n-2} & \sim K_{j-i} F \otimes C H^{i} X_{n} &
\end{array}
$$

commutes for $i \leq j$. Inductively we get the commutative diagrams

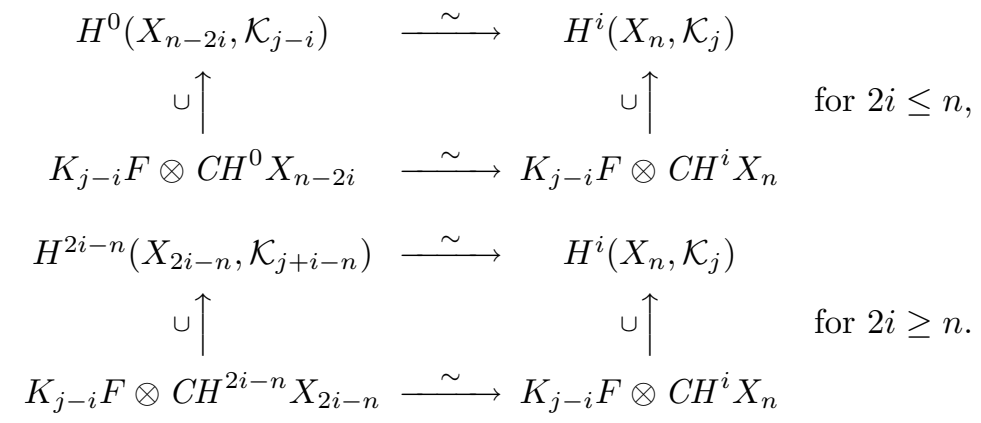

Still by lemma $2.1, K_{j-i} F \otimes C H^{0} X_{n-2 i} \stackrel{\cup}{\longrightarrow} H^{0}\left(X_{n-2 i}, \mathcal{K}_{j-i}\right)$ and

$$
K_{j-i} F \otimes C H^{2 i-n} X_{2 i-n} \stackrel{\cup}{\longrightarrow} H^{2 i-n}\left(X_{2 i-n}, \mathcal{K}_{j+i-n}\right)
$$

are isomorphisms (note that for $2 i=n, X_{0}$ is a disjoint union of two points so $\left.C H^{0} X_{0} \simeq \mathbf{Z} \oplus \mathbf{Z}\right)$. The claim follows.

Lemma 2.3. Let $i>0$ and $X$ be a quadric over $F$, with $\operatorname{dim} X \neq 2 i$ or $\operatorname{dim} X=2 i$ and $d(X)=1$. Then, for all $j \geq i$, the map $K_{j-i} F \otimes C H^{i} X \rightarrow H^{i}\left(X, \mathcal{K}_{j}\right)$ has 2-primary torsion kernel and cokernel. If $\operatorname{dim} X=2 i$ and $d(X)=d \neq 1$, the composition

$$
\left(K_{j-i} E \otimes C H^{i} X_{E}\right)_{\Gamma} \rightarrow H^{i}\left(X_{E}, \mathcal{K}_{j}\right)_{\Gamma} \stackrel{N}{\longrightarrow} H^{i}\left(X, \mathcal{K}_{j}\right)
$$


has 2-primary torsion kernel and cokernel, where $E=F(\sqrt{d}), \Gamma=\operatorname{Gal}(E / F)$ and $N$ is the transfer in $\mathcal{K}$-cohomology.

Proof. Assume first that $\operatorname{dim} X \neq 2 i$ or $\operatorname{dim} X=2 i$ and $d(X)=1$. Let $K$ be a splitting field for $X$ that is finite and Galois over $F$, with $[K: F]$ a power of 2 (some multiquadratic extension will do) and $G=\operatorname{Gal}(K / F)$. In the commutative diagram

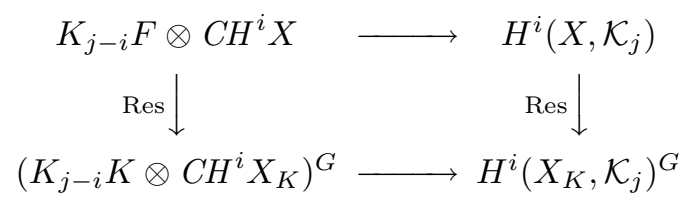

the bottom horizontal map is an isomorphism by proposition 2.2 and the right vertical map has 2-primary torsion kernel by the usual transfer argument. Therefore, to prove the claim, it is enough to show that the left vertical map has 2-primary torsion kernel and cokernel. But this map decomposes as

$$
\begin{aligned}
K_{j-i} F \otimes C H^{i} X \rightarrow K_{j-i} F \otimes & C H^{i} X_{K} \rightarrow \\
& \rightarrow\left(K_{j-i} K\right)^{G} \otimes C H^{i} X_{K}=\left(K_{j-i} K \otimes C H^{i} X_{K}\right)^{G}
\end{aligned}
$$

since $C H^{i} X_{K} \simeq \mathbf{Z}$ or $\mathbf{Z} \oplus \mathbf{Z}$ with trivial Galois action by propositions 1.1 and 2.2. The same proposition shows that the left map has 2-primary torsion kernel and cokernel, while the transfer argument shows the same for the right map. This implies the same for their composition.

Assume now that $\operatorname{dim} X=2 i$ and $d(X)=d \neq 1$. By the above, the left map in the composition of lemma 2.3 has 2-primary torsion kernel and cokernel, and the same holds for the right one still by a transfer argument. This concludes the proof of lemma 2.3 .

Proposition 2.4. Let $i>0$ and $X$ be a quadric over $F$, with $\operatorname{dim} X \neq 2 i-2$ or $\operatorname{dim} X=2 i-2$ and $d(X)=1$. Then there is an exact sequence:

$$
H^{1}(F, 1) \otimes C H^{i-1} X \rightarrow H^{i-1}\left(X, \mathcal{H}^{i}(i)\right) \rightarrow\left(C H^{i} X\right)_{\text {torsion }} \rightarrow 0 .
$$

If $\operatorname{dim} X=2 i-2$ and $d(X)=d \neq 1$, there is an exact sequence:

$$
\left(H^{1}(E, 1) \otimes C H^{i-1} X_{E}\right)_{\Gamma} \rightarrow H^{i-1}\left(X, \mathcal{H}^{i}(i)\right) \rightarrow\left(C H^{i} X\right)_{\text {torsion }} \rightarrow 0,
$$

where $E=F(\sqrt{d}), \Gamma=\operatorname{Gal}(E / F)$ and the left map is cup-product followed by transfer.

Proof. Tensoring the maps of lemma 2.3 with $\mathbf{Q} / \mathbf{Z}$ we get surjections:

$$
K_{1} F \otimes C H^{i-1} X \otimes \mathbf{Q} / \mathbf{Z} \longrightarrow H^{i-1}\left(X, \mathcal{K}_{i}\right) \otimes \mathbf{Q} / \mathbf{Z}
$$

if $\operatorname{dim} X \neq 2 i-2$ or $\operatorname{dim} X=2 i-2$ and $d(X)=1$,

$$
\begin{aligned}
&\left(K_{1} E \otimes C H^{i-1} X_{E} \otimes \mathbf{Q} / \mathbf{Z}\right)_{\Gamma}=\left(K_{1} E \otimes C H^{i-1} X_{E}\right)_{\Gamma} \otimes \mathbf{Q} / \mathbf{Z} \\
& \longrightarrow H^{i-1}\left(X, \mathcal{K}_{i}\right) \otimes \mathbf{Q} / \mathbf{Z}
\end{aligned}
$$

if $\operatorname{dim} X=2 i-2$ and $d(X)=d \neq 1$.

By Kummer theory, $K_{1} F \otimes \mathbf{Q} / \mathbf{Z} \simeq H^{1}(F, 1)$. The claim now follows from the short exact sequence $[5,3.6]$

$$
0 \rightarrow H^{i-1}\left(X, \mathcal{K}_{i}\right) \otimes \mathbf{Q} / \mathbf{Z} \rightarrow H^{i-1}\left(X, \mathcal{H}^{i}(i)\right) \rightarrow\left(C H^{i} X\right)_{\text {torsion }} \rightarrow 0 .
$$




\section{Proposition 2.5.}

a) ([8, prop. 1.2]) If the quadric $X$ is isotropic over $F$, then $\eta_{2}^{i}$ and $\eta^{i}$ are isomorphisms for all $i \geq 0$.

b) In general, Ker $\eta^{i}$ and Coker $\eta^{i}$ have exponent $\leq 2$.

c) (cf. $\left[10\right.$, lemma 1.3]) Suppose $X$ and $X^{\prime}$ are two quadrics such that $X^{\prime}$ becomes isotropic over $F(X)$. Then there is a natural commutative diagram for all $i, k$ :

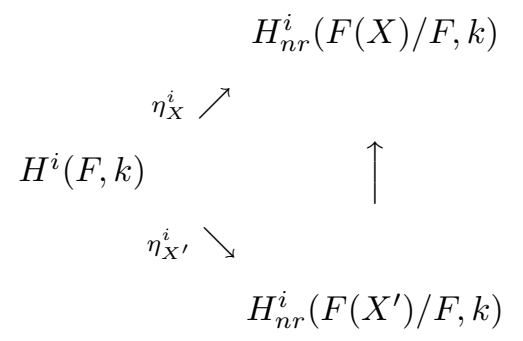

so that $\operatorname{Ker} \eta_{X^{\prime}}^{i} \subseteq \operatorname{Ker} \eta_{X}^{i}$ and Coker $\eta_{X^{\prime}}^{i}$ maps to Coker $\eta_{X}^{i}$. If $X$ also becomes isotropic over $F\left(X^{\prime}\right)$, then $\operatorname{Ker} \eta_{X}^{i}=\operatorname{Ker} \eta_{X^{\prime}}^{i}$ and Coker $\eta_{X}^{i} \simeq$ Coker $\eta_{X^{\prime}}^{i}$.

Proof. a) comes from the fact that the extension $F(X) / F$ is purely transcendental if $X$ is isotropic (compare [7, th. 4.1.5]). b) follows from a) and a transfer argument, using a quadratic extension of $F$ which makes $X$ isotropic. We give a proof of c) which is more elementary than [10, lemma 1.3]. Consider the diagram of fields

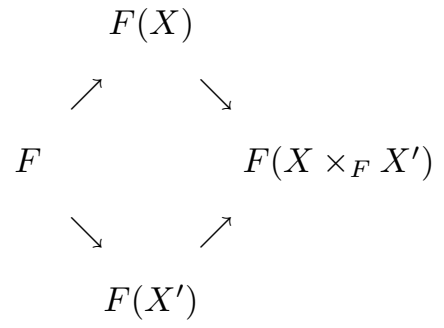

There are natural maps

$$
H_{n r}^{i}(F(X) / F, k) \rightarrow H_{n r}^{i}\left(F\left(X \times_{F} X^{\prime}\right) / F, k\right)
$$

and

$$
H_{n r}^{i}\left(F\left(X^{\prime}\right) / F, k\right) \rightarrow H_{n r}^{i}\left(F\left(X \times{ }_{F} X^{\prime}\right) / F, k\right) .
$$

If $X^{\prime}$ becomes isotropic over $F(X)$, then $F\left(X \times_{F} X^{\prime}\right) / F(X)$ is a purely transcendental extension. Therefore, by [8, prop. 1.2] or [7, th. 4.1.5], the first quoted map is an isomorphism, which proves the first claim. If $X$ is also purely transcendental over $F\left(X^{\prime}\right)$, then $H_{n r}^{i}\left(F\left(X^{\prime}\right) / F, k\right) \rightarrow H_{n r}^{i}(F(X) / F, k)$ is clearly the inverse of $H_{n r}^{i}(F(X) / F, k) \rightarrow H_{n r}^{i}\left(F\left(X^{\prime}\right) / F, k\right)$, hence the second claim.

\section{Part II. Ker $\eta_{2}^{4}$ for higher-dimensional quadrics}

\section{Generalities on $\operatorname{Ker} \eta_{2}^{i}$}

We fix a number $i$ and assume that the invariant $\bar{e}_{F}^{r}$ is is well-defined for $r \leq i-1$ and an isomorphism for $r \leq i-2$ (for all fields $F$ ).

Lemma 3.1. A symbol $\left(a_{1}, \ldots, a_{i}\right) \neq 0$ lies in $\operatorname{Ker} \eta_{2}^{i}$ if and only if $q$ is similar to a subform of $\left\langle\left\langle a_{1}, \ldots, a_{i}\right\rangle\right\rangle$. 
Proof. Let $\varphi=\left\langle\left\langle a_{1}, \ldots, a_{i}\right\rangle\right\rangle$. Since $\left(a_{1}, \ldots, a_{i}\right)_{F(X)}=0$, [16, remark p. 555] implies $\varphi_{F(X)} \sim 0$, and the claim follows by the Cassels-Pfister theorem [26, th. IX.1.3].

As observed in the introduction, for $\operatorname{dim} q \leq 2^{i-2}$ the group Ker $\eta_{2}^{i}$ does not consist of symbols in general. The following proposition and corollary indicate that this bound on $\operatorname{dim} q$ might be sharp. Recall that two $n$-fold Pfister forms $\varphi, \psi$ are linked if there exist $a_{1}, \ldots, a_{n-1}, a_{n}, b_{n} \in F^{*}$ such that $\varphi \simeq\left\langle\left\langle a_{1}, \ldots, a_{n-1}, a_{n}\right\rangle\right\rangle$ and $\psi \simeq\left\langle\left\langle a_{1}, \ldots, a_{n-1}, b_{n}\right\rangle\right\rangle$. We define the notion of linked symbols (in Galois cohomology) similarly.

Proposition 3.2. Let $\operatorname{dim} q \geq 2^{i-2}+1$, let $X$ be the projective quadric with equation $q=0$ and let $\varphi, \psi \in \operatorname{Ker}(W(F) \rightarrow W(F(X)))$ be two $i$-fold Pfister forms. Then $\varphi$ and $\psi$ are linked, hence $\varphi \perp-\psi$ is Witt-equivalent to a third $i$-fold Pfister form (up to similarity).

Proof. (cf. Fitzgerald [15, proof of th. 2.1]) The forms $\varphi$ and $\psi$ are multiplicative and split over $F(X)$. Therefore $q$ is isomorphic to subforms of $\varphi$ and of $\psi$ (assuming $q$ represents 1). Hence the split form $q \perp-q$ is a subform of $\varphi \perp-\psi$ and the Witt index $n=i(\varphi \perp-\psi)$ is $\geq 2^{i-2}+1$. By [13, th. 4.5], $n$ is a power of 2 , hence $n \geq 2^{i-1}$. Therefore $\varphi$ and $\psi$ are linked, again by loc. cit..

Lemma 3.3. For $\operatorname{dim} q \geq 2^{i-2}+1$ the following are equivalent:

a) Ker $\eta_{2}^{i}$ consists of symbols;

b) $\operatorname{Ker} \eta_{2}^{i}$ is additively generated by symbols.

Proof. Let $\alpha, \beta \in \operatorname{Ker} \eta_{2}^{i}$ be two symbols and $\tilde{\alpha}, \tilde{\beta}$ Pfister forms such that $e^{i}(\tilde{\alpha})=$ $\alpha, e^{i}(\tilde{\beta})=\beta$. By [16, remark p. 555], $\tilde{\alpha}, \tilde{\beta} \in \operatorname{Ker}(W(F) \rightarrow W(F(X))$. By proposition 3.2, $\tilde{\alpha}$ and $\tilde{\beta}$ are linked, so $\alpha$ and $\beta$ are linked and $\alpha+\beta$ is a symbol.

Proposition 3.4. Assume that for $\operatorname{dim} q=2^{i-2}+1$ the group Ker $\eta_{2}^{i}$ consists of symbols. Then

(1) Ker $\eta_{2}^{i}$ consists of symbols for $\operatorname{dim} q>2^{i-2}$.

(2) $\left|\operatorname{Ker} \eta_{2}^{i}\right| \leq 2$ for $\operatorname{dim} q>2^{i-1}$.

(3) $\operatorname{Ker} \eta_{2}^{i}=0$ for $\operatorname{dim} q>2^{i}$.

Remark. The hypothesis of proposition 3.4 is satisfied for $i \leq 3$ by [1]. For $i=4$ it will be proved in the next three sections (theorem 6.3).

Proof. (1) Let $\operatorname{dim} q^{\prime} \geq 2^{i-2}+1$ and let $q$ be a subform of $q^{\prime}$ of dimension $2^{i-2}+1$. The claim follows now from proposition $2.5 \mathrm{c}$ ) applied to the associated quadrics $X^{\prime}$ resp. $X$.

$(2)+(3)$ If $\operatorname{dim} q>2^{i-1}$, for any nonzero symbol $\left(a_{1}, \ldots, a_{i}\right) \in \operatorname{Ker} \eta_{2}^{i}, q$ is a neighbour of the Pfister form $\left\langle\left\langle a_{1}, \ldots, a_{i}\right\rangle\right\rangle$ by lemma 3.1. But $q$ can be a neighbour of at most one Pfister form, up to isomorphism, $c f .[24,7.4]$. Moreover if $\left\langle\left\langle a_{1}, \ldots, a_{i}\right\rangle\right\rangle \simeq\left\langle\left\langle b_{1}, \ldots, b_{i}\right\rangle\right\rangle$, then $\left(a_{1}, \ldots, a_{i}\right)=\left(b_{1}, \ldots, b_{i}\right)[1$, Satz 1.6]. Hence $\left|\operatorname{Ker} \eta_{2}^{i}\right| \leq 2$, and if it is nontrivial then $\operatorname{dim} q \leq 2^{i}$.

\section{6-dimensional spinors}

For generalities on Clifford algebras and the special Clifford group see [45].

Let $q: V \rightarrow F$ be 6-dimensional, let $C_{0}(q)$ be the even Clifford algebra of $q$, and let $Z(q)$ be the center of $C_{0}(q)$. Then $Z(q)$ is a quadratic extension of $F$ and $C_{0}(q)$ 
is an Azumaya algebra of degree 4 over $Z(q)$. The standard involution $x \mapsto x^{t}$ is an involution of second kind. Denote by

$$
\text { Nrd: } C_{0}(q) \rightarrow Z(q)
$$

the reduced norm.

Let

$$
\mathrm{S} \Gamma(q)=\left\{x \in C_{0}(q)^{*} \mid x V x^{-1}=V\right\}
$$

be the special Clifford group and let

$$
\mathrm{G} \Gamma(q)=\left\{x \in C_{0}(q)^{*} \mid x x^{t} \in F^{*}\right\} .
$$

Then $\mathrm{S} \Gamma(q) \subset \mathrm{G} \Gamma(q)$ and there is an exact sequence

$$
1 \rightarrow \operatorname{Spin}(q) \rightarrow \mathrm{S} \Gamma(q) \stackrel{\text { sn }}{\longrightarrow} F^{*}
$$

where $\operatorname{sn}(x)=x x^{t}$ is the spinor norm.

Suppose $d_{ \pm}(q)=1$. Then $Z(q)=F \times F$ and $C_{0}(q)=A \times A^{\prime}$ where $A$ and $A^{\prime}$ are algebras of degree 4 over $F$. The standard involution identifies $A^{\prime}$ with $A^{\text {op }}$, so that there is an isomorphism

$$
C_{0}(q)=A \times A^{\mathrm{op}}
$$

under which the standard involution reads as $(a, b)^{t}=(b, a)$. The reduced norm $C_{0}(q) \rightarrow Z(q)$ is then componentwise the reduced norm on $A$. The group GГ $(q)$ becomes the group of pairs

$$
\left\{\left(a, a^{-1} f\right) \in A \times A^{\mathrm{op}} \mid a \in A^{*}, f \in F^{*}\right\} .
$$

Let $\pi: C_{0}(q) \rightarrow A$ be the projection onto the first factor. Then

$$
\mathrm{G} \Gamma(q)=A^{*} \times F^{*}
$$

via $x \mapsto\left(\pi(x), x x^{t}\right)$.

We call an element $x \in \mathrm{S} \Gamma(q)$ plane if $x \in \mathrm{S} \Gamma(\tilde{q})$ where $\tilde{q}$ is a regular 2dimensional subform of $q$. If $q$ is anisotropic, then $x$ is plane if and only if $x$ is the product $v w$ of two vectors $v, w \in V \backslash 0$.

Lemma 4.1. $\operatorname{Nrd}(x)=\left(x x^{t}\right)^{2}$ for $x \in \mathrm{S} \Gamma(q)$.

Proof. The action of $\mathrm{S} \Gamma(q)$ on $V$ induces an exact sequence

$$
1 \rightarrow F^{*} \rightarrow \mathrm{S} \Gamma(q) \rightarrow \mathrm{SO}(q) \rightarrow 1 .
$$

Since $\mathrm{O}(q)$ is generated by reflections, it follows that $\mathrm{S} \Gamma(q)$ is generated by products $x=v w$ where $v, w \in V$ are anisotropic vectors. For generic $v, w$, the subspace of $V$ generated by $v, w$ is regular of dimension 2 . Therefore it suffices to check the claim for plane elements.

So suppose $x \in C_{0}(\tilde{q})$ for a regular 2-dimensional subform $\tilde{q}$. Write $L=C_{0}(\tilde{q})$. The standard involution leaves $L$ invariant and restricts to the canonical involution of $L / F$. Hence $x \mapsto x x^{t}$ restricts on $L$ to the norm of $L / F$. The claim follows now from the fact that $C_{0}(q)$ is of degree 4 and $L \otimes Z(q) \subset C_{0}(q)$ is of degree 2 over $Z(q)$.

\section{Proposition 4.2.}

a) $\mathrm{S} \Gamma(q)=\left\{x \in C_{0}(q)^{*} \mid x x^{t} \in F^{*}, \operatorname{Nrd}(x)=\left(x x^{t}\right)^{2}\right\}$,

b) $\operatorname{Spin}(q)=\left\{x \in C_{0}(q)^{*} \mid x x^{t}=1, \operatorname{Nrd}(x)=1\right\}$. 
Proof. The inclusions " $\subset$ " are clear from lemma 4.1.

We may assume that $F$ is algebraically closed. In this case it easy to see that $\mathrm{S} \Gamma(q)=\operatorname{Spin}(q) \cdot F^{*}$ and similarly for the righthand sides. Hence b) $\Rightarrow$ a).

The righthand side of b) becomes under (4.1) the group of pairs

$$
\left\{\left(a, a^{-1}\right) \in A \times A^{\text {op }} \mid a \in A^{*}, \operatorname{Nrd}(a)=1\right\}=\operatorname{SL}(1, A) .
$$

This way b) reads as the classical isomorphism $\operatorname{Spin}(6)=\operatorname{SL}(4)[55,12,25]$.

Corollary 4.3. If $d_{ \pm}(q)=1$ and $A$ is as above, there is an exact sequence

$$
1 \rightarrow \mathrm{S} \Gamma(q) \rightarrow A^{*} \times F^{*} \stackrel{\omega}{\longrightarrow} F^{*}
$$

where $\omega(a, f)=\operatorname{Nrd}(a) / f^{2}$.

Corollary 4.4. Let $A$ be a biquaternion skewfield and let $a \in A^{*}$ and $f \in F^{*}$ with

$$
\operatorname{Nrd}(a)=f^{2} .
$$

Then there exist $a_{1}, a_{2}, a_{3} \in A^{*}$ with the following properties:

- $a=a_{1} a_{2} a_{3}$,

- Every $a_{i}$ lies in a quadratic subfield $L_{i} \subset A$,

- $N_{L_{1} / F}\left(a_{1}\right) N_{L_{2} / F}\left(a_{2}\right) N_{L_{3} / F}\left(a_{3}\right)=f$.

Proof. If $A=(a, b) \otimes(c, d)$, let $q=\langle-a,-b, a b, c, d,-c d\rangle$. We are then in the situation of corollary 4.3. The form $q$ is anisotropic. Since every element in $\operatorname{SO}(q)$ is a product of 6 reflections, it follows that every element in $\mathrm{S} \Gamma(q)$ is a product of 3 plane elements $x_{i}$. Their images $a_{i}=\pi\left(x_{i}\right)$ in $A^{*}$ do the job.

We will have to make use of the following variant of the Cartan-Dieudonné theorem for anisotropic forms.

Lemma 4.5. Let $q: V \rightarrow F$ be anisotropic of dimension $n$ and let $V_{1} \subset V_{2} \subset$ $\cdots \subset V_{n}=V$ be a sequence of subspaces with $\operatorname{dim} V_{i}=i$. Then every $\alpha \in \mathrm{O}(q)$ is a product

$$
\alpha=\tau_{i_{k}} \cdots \tau_{i_{1}}
$$

where $1 \leq i_{1}<\cdots<i_{k} \leq n$ and $\tau_{j}$ is a reflection in $\mathrm{O}\left(q \mid V_{j}\right)$.

Proof. By induction on $n$. Let $v \neq 0$ be orthogonal to $V_{n-1}$. If $\alpha \notin \mathrm{O}\left(q \mid V_{n-1}\right)$, let $\tau$ be the reflection at the hyperplane orthogonal to $\alpha(v)-v$. Then $\tau \alpha \in$ $\mathrm{O}\left(q \mid V_{n-1}\right)$.

\section{5. $\operatorname{Ker} \eta_{2}^{4}$ and $H^{2}\left(X, \mathcal{K}_{3}\right)$}

The following theorem reduces the computation of $\operatorname{Ker} \eta_{2}^{4}$ for $\operatorname{dim} q \geq 5$ to a $\mathcal{K}$ cohomology computation. The statement is essentially a reformulation of arguments in [27], where the result has been applied to Albert forms. The principal idea has already been used in [16] for 5-dimensional Pfister neighbours.

Theorem 5.1. Let $X$ be a smooth quadric of dimension $\geq 3$. Then there exist isomorphisms

$$
\begin{aligned}
& \rho_{3}: \operatorname{Ker}\left(H^{2}\left(X, \mathcal{K}_{2}\right) \rightarrow H^{2}\left(\bar{X}, \mathcal{K}_{2}\right)\right) \stackrel{\sim}{\rightarrow} \operatorname{Ker}\left(H^{3} F \rightarrow H^{3} F(X)\right), \\
& \rho_{4}: \operatorname{Ker}\left(H^{2}\left(X, \mathcal{K}_{3}\right) \rightarrow H^{2}\left(\bar{X}, \mathcal{K}_{3}\right)\right) \stackrel{\sim}{\longrightarrow} \operatorname{Ker}\left(H^{4} F \rightarrow H^{4} F(X)\right) .
\end{aligned}
$$

These are functorial with respect to base change $F \rightarrow F^{\prime}$ and with respect to transfer for finite extensions $F^{\prime} / F$. 
Moreover they commute with multiplication with $K_{1}$ in the following sense:

$$
\rho_{4}(\{a\} \cdot x)=(a) \cdot \rho_{3}(x)
$$

for $a \in F^{*}$ and $x \in \operatorname{Ker}\left(H^{2}\left(X, \mathcal{K}_{2}\right) \rightarrow H^{2}\left(\bar{X}, \mathcal{K}_{2}\right)\right)$.

Proof. The (inverse of the) map $\rho_{4}$ has been described in [27, p. 71] in terms of a diagram chase. It is also proven that the map is an isomorphism (it should be noted that condition (7) in [27, p. 71] is superfluous). From this definition the compatibility with respect to base change and transfer is easy to see.

One proceeds in a similar way for $\rho_{3}$ (see also [16, diagrams (3.5), (4.2)]), just by repeating the diagram chase in one degree less. The product formula is then obvious.

Remark. It can be shown that the maps $\rho_{i}$ are functorial with respect to linear morphisms $j: X \subset X^{\prime}$ of quadrics. Namely then $\operatorname{Ker} \eta_{X^{\prime}}^{i} \subset \operatorname{Ker} \eta_{X}^{i}$ (proposition $2.5 \mathrm{c}$ )) and the maps $\rho_{i}$ commute with these inclusions and with the pull back maps $j^{*}$ on $H^{2}\left(-, \mathcal{K}_{i-1}\right)$.

Our aim is to compute the map $\rho_{4}$ for $\operatorname{dim} X=3$.

Let $q$ be a form defining $X$ and let $A=C_{0}(q)$ be its even Clifford algebra (a biquaternion algebra). We denote by $h \in C H^{1}(X)$ the class of a hyperplane section. If $A$ is split, then $X$ is split. In this case $C H^{2}(X)=\mathbf{Z}$, generated by the class $l$ of a line and one has $h^{2}=2 l$.

Theorem 5.2. For $\operatorname{dim} X=3$ there exist surjections

$$
\begin{aligned}
& \theta_{3}: K_{0} A \oplus K_{0} F \rightarrow H^{2}\left(X, \mathcal{K}_{2}\right), \\
& \theta_{4}: K_{1} A \oplus K_{1} F \rightarrow H^{2}\left(X, \mathcal{K}_{3}\right),
\end{aligned}
$$

functorial with respect to base change and transfer, compatible with multiplication with $K_{1}$, and such that $\theta_{3}$ has the following properties:

a) $\theta_{3}(0,1)=-h^{2}$.

b) If $A$ is split, let $y \in K_{0} A$ be the element with $\operatorname{Nrd}(y)=1$. Then $\theta_{3}(y, 0)=l$.

Proof. All the essential arguments are in [27, p. 74-76]. In fact, in the 5-dimensional case the situation is somewhat simpler. By [27, prop. 2],

$$
H^{2}\left(X, \mathcal{K}_{3}\right)=K_{1}(X)^{(2)} / K_{1}(X)^{(3)}
$$

where the superscripts refer to the filtration coming from the Brown-GerstenQuillen (BGQ) spectral sequence [41]. In our case one finds $K_{1}(X)^{(2)}=K_{1} A \oplus K_{1} F$. (We don't have to care about a computation of $\left.K_{1}(X)^{(3)}\right)$.

Let

$$
\operatorname{Res}_{\bar{F} / F}: H^{2}\left(X, \mathcal{K}_{i}\right) \rightarrow H^{2}\left(\bar{X}, \mathcal{K}_{i}\right)
$$

be the restriction to a separable closure. Theorem $5.2 \mathrm{a}$ ), b) shows

$$
\operatorname{Res}_{\bar{F} / F} \circ \theta_{3}(y, m)=(\operatorname{Nrd}(y)-2 m) \cdot l \in C H^{2}(\bar{X})=\mathbf{Z} \cdot l .
$$

The compatibility of $\theta_{3}, \theta_{4}$ with multiplication with $K_{1}$ yields

$$
\operatorname{Res}_{\bar{F} / F} \circ \theta_{4}(z,\{f\})=(\operatorname{Nrd}(z)-2\{f\}) \cdot l \in H^{2}\left(\bar{X}, \mathcal{K}_{3}\right)=K_{1} \bar{F} \cdot l .
$$

This together with theorem 5.1 and theorem 5.2 yields 
Corollary 5.3. There are surjections

$$
\begin{aligned}
& \omega_{3}: \operatorname{Ker}\left(K_{0} A \oplus K_{0} F \stackrel{(\mathrm{Nrd},-2)}{\longrightarrow} K_{0} F\right) \rightarrow \operatorname{Ker} \eta_{2}^{3}, \\
& \omega_{4}: \operatorname{Ker}\left(K_{1} A \oplus K_{1} F \stackrel{(\mathrm{Nrd},-2)}{\longrightarrow} K_{1} F\right) \rightarrow \operatorname{Ker} \eta_{2}^{4},
\end{aligned}
$$

compatible with base change, transfer and multiplication with $K_{1}$.

\section{Computations for $\operatorname{dim} X=3$}

Let $q: W \rightarrow F$ be 5 -dimensional and $A=C_{0}(q)$. We assume that the determinant of $q$ is -1 . Put

$$
\begin{gathered}
\widehat{q}: F \oplus W \rightarrow F \\
\widehat{q}(t, w)=t^{2}+q(w) .
\end{gathered}
$$

Then $C_{0}(\widehat{q})=A \times A^{\text {op }}$ as in (4.1).

We first compute $\omega_{3}, \omega_{4}$ in the case when $\widehat{q}$ is isotropic. In this case $q$ represents -1 and therefore

$$
q \simeq\langle-1\rangle \perp c\langle\langle a, b\rangle\rangle
$$

for some $a, b, c \in F^{*}$. Moreover $A=M_{2}(F) \otimes D$ where $D$ is the quaternion algebra associated to $a, b$.

Proposition 6.1. Let $y \in K_{0} A=K_{0} D$ be the element represented by the $D$ module $D$ (i.e., $\operatorname{Nrd}(y)=2$ ) and let $\varepsilon=(y, 1)$.

(1) The sequences

$$
\begin{aligned}
& K_{0} F \stackrel{\cdot \varepsilon}{\longrightarrow} K_{0} A \oplus K_{0} F \stackrel{(\mathrm{Nrd},-2)}{\longrightarrow} K_{0} F, \\
& K_{1} F \stackrel{\cdot \varepsilon}{\longrightarrow} K_{1} A \oplus K_{1} F \stackrel{(\mathrm{Nrd},-2)}{\longrightarrow} K_{1} F
\end{aligned}
$$

are exact.

(2) One has

$$
\begin{aligned}
\omega_{3}(\varepsilon) & =(a, b, c), \\
\omega_{4}(\{e\} \cdot \varepsilon) & =(e, a, b, c), \quad e \in F^{*} .
\end{aligned}
$$

Proof. (1) follows from the injectivity of the reduced norm $K_{i} D \rightarrow K_{i} F$ for $i=0,1$.

Corollary 5.3 shows that $\omega_{3}(\varepsilon)$ generates $\operatorname{Ker} \eta_{2}^{3}$. Since $(a, b, c) \in \operatorname{Ker} \eta_{2}^{3}$, we must have the first formula in (2). (If $(a, b, c)=0$, then $q$ is isotropic and Ker $\eta_{2}^{3}=0$.) The second formula follows from the compatibility with multiplication.

We now assume that $\widehat{q}$ is anisotropic. Via corollary 4.3, the map $\omega_{4}$ of corollary 5.3 reads as a map

$$
\tilde{\omega}_{4}: \mathrm{S} \Gamma(\widehat{q}) \rightarrow \operatorname{Ker} \eta_{2}^{4} .
$$

We compute this map for plane elements $x \in \mathrm{S} \Gamma(\widehat{q})$.

Suppose $x \in L=C_{0}(\tilde{q})$ for a 2-dimensional subform $\tilde{q}$ of $\widehat{q}$. The map

$$
L^{*}=C_{0}(\tilde{q})^{*}=\mathrm{S} \Gamma(\tilde{q}) \subset \mathrm{S} \Gamma(\widehat{q}) \rightarrow A^{*} \times F^{*}
$$

coincides with the map

$$
K_{1} L \rightarrow K_{1} A \oplus K_{1} F
$$

given by the inclusion $L \subset A$ and the norm for $L / F$. 
The form $\widehat{q}_{L}$ is isotropic. Denote by

$$
\varepsilon_{L} \in \operatorname{Ker}\left(K_{0}(A \otimes L) \oplus K_{0} L \stackrel{(\mathrm{Nrd},-2)}{\longrightarrow} K_{0} L\right)
$$

the generator considered above.

By compatibility with transfer for $L / F$ one finds

$$
\tilde{\omega}_{4}(x)=\operatorname{Cor}_{L / F}\left((x) \cdot \omega_{3}\left(\varepsilon_{L}\right)\right)
$$

Note that if $\tilde{q}$ is a subform of $q$, then $q_{L}$ is isotropic and therefore $\omega_{3}\left(\varepsilon_{L}\right)=0$.

We compute $\omega_{4}(u)$ for arbitrary $u \in \mathrm{S} \Gamma(\widehat{q})$. If $u \in \mathrm{S} \Gamma(q)$, then $u$ is a product of plane elements in $\mathrm{S} \Gamma(q)$ and $\tilde{\omega}_{4}(u)=0$ by the last remark. In general we have

Lemma 6.2. For any $u \in S \Gamma(q)$, there exist a one-dimensional subspace $H$ of $W$ and a plane element $x \in S \Gamma(\hat{q} \mid(F \oplus H))$ such that $u \in x \cdot S \Gamma(q)$.

Proof. There exist by lemma 4.5 and the exact sequence (4.2) a vector $v_{1} \in F \oplus W$ and vectors $v_{2}, \ldots, v_{6} \in W$ such that $u=v_{1} \cdots v_{6}$.

Write $v_{1}=s(1,0)+t h$ with $s, t \in F^{*}, 0 \neq h \in W$, and let $H$ be the subspace generated by $h$. Then $x=v_{1} h$ has the desired properties.

Since $\omega_{4} \mid \mathrm{S} \Gamma(q)=0$, we are reduced to computing $\tilde{\omega}_{4}$ on plane elements $x \in$ $S \Gamma(\hat{q} \mid F \oplus H)$ as in lemma 6.2. Let $h$ be a basis vector of $H$ and let $a=-q(h)$. Then $L=C_{0}(\tilde{q})=F(\sqrt{a})$ and $q$ is of the form

$$
q \simeq\left\langle-a, a_{1}, a_{2}, a_{3}, a a_{1} a_{2} a_{3}\right\rangle
$$

Over $L$ this gives

$$
q_{L} \simeq\left\langle-1, a_{1}, a_{2}, a_{3}, a_{1} a_{2} a_{3}\right\rangle \simeq\langle-1\rangle \perp a_{1}\left\langle\left\langle-a_{1} a_{2},-a_{1} a_{3}\right\rangle\right\rangle .
$$

Hence

$$
\omega_{3}\left(\varepsilon_{L}\right)=\operatorname{Res}_{L / F}\left(\left(a_{1},-a_{1} a_{2},-a_{1} a_{3}\right)\right)
$$

comes from the ground field (although $\varepsilon_{L}$ does not). So

$$
\tilde{\omega}_{4}(x)=\operatorname{Cor}_{L / F}\left((x) \cdot \omega_{3}\left(\varepsilon_{L}\right)\right)=\left(N_{L / F}(x), a_{1},-a_{1} a_{2},-a_{1} a_{3}\right)
$$

is a symbol.

We have proved:

Theorem 6.3. If $\operatorname{dim} X=3$, then $\operatorname{Ker} \eta_{2}^{4}$ consists of symbols.

Remark. The homomorphism $\tilde{\omega}_{4}$ is obviously trivial on the commutator subgroup $[\mathrm{S} \Gamma(\widehat{q}), \mathrm{S} \Gamma(\widehat{q})]$. Moreover it is easy to see that the map

$$
\mathrm{S} \Gamma(\widehat{q}) / \mathrm{S} \Gamma(q) \rightarrow \mathrm{O}(\widehat{q}) / \mathrm{O}(q)
$$

is bijective. Hence the map $\tilde{\omega}_{4}$ actually induces a surjective homomorphism

$$
\Omega: \mathrm{O}(\widehat{q}) /[\mathrm{O}(\widehat{q}), \mathrm{O}(\widehat{q})] \mathrm{O}(q) \rightarrow \operatorname{ker}\left(H^{4} F \rightarrow H^{4} F(X)\right)
$$

Merkurjev and Tignol have described this map in terms of symplectic involutions $[28,52]$.

The kernel of the spinor norm

$$
\mathrm{O}(\widehat{q}) /[\mathrm{O}(\widehat{q}), \mathrm{O}(\widehat{q})] \rightarrow F^{*} /\left(F^{*}\right)^{2}
$$

can be identified with $S K_{1} A$. The restriction of $\Omega$ to $S K_{1} A$ depends only on $\widehat{q}$ (and not on the choice of 5 -dimensional subform $q$ ). It fits into an exact sequence [27]

$$
0 \rightarrow S K_{1} A \stackrel{\Omega}{\longrightarrow} H^{4} F \rightarrow H^{4} F(\widehat{X})
$$


where $\widehat{X}$ is the quadric for $\widehat{q}$.

Further details will appear in [25].

\section{Part III. Coker $\eta_{2}^{i}$ for higher-dimensional quadrics}

\section{A relationship between $\operatorname{Ker} \boldsymbol{\eta}_{2}^{i}$ and Coker $\eta_{2}^{i}$}

In this section, we prove a general result which we then apply to quadrics. To start with, let $F$ have arbitrary characteristic exponent $p$ and $X$ be a smooth variety over $F$. We denote by $\delta=\delta(X / F)$ the prime-to- $p$ part of the $g c d$ of degrees of closed points of $X$. Let $n$ be some integer divisible by $\delta$ and prime to $p, i \geq 1$ and let

$$
\begin{aligned}
\eta_{n}^{i}: H^{i}\left(F, \mu_{n}^{\otimes(i-1)}\right) & \rightarrow H^{0}\left(X, \mathcal{H}^{i}\left(\mu_{n}^{\otimes(i-1)}\right)\right) \\
\eta^{i}: H^{i}(F, i-1) & \rightarrow H^{0}\left(X, \mathcal{H}^{i}(i-1)\right)
\end{aligned}
$$

be the natural maps.

Proposition 7.1. Let $m$ be another integer divisible by $\delta$ and prime to $p$. For $i \leq 3$, there is an exact sequence

$$
0 \rightarrow \operatorname{Ker} \eta_{m}^{i} \cap \operatorname{Im}\left(H^{i}\left(F, \mu_{m n}^{\otimes(i-1)}\right) \stackrel{n}{\rightarrow} H^{i}\left(F, \mu_{m}^{\otimes(i-1)}\right)\right) \rightarrow \text { Coker } \eta_{n}^{i} \rightarrow \text { Coker } \eta^{i}
$$

If $m, n$ are powers of 2 , this result also holds for $i=4$.

Proof. We shall freely use the following fact, which is a consequence of Kummer theory for $i=2$ (resp. the Merkurjev-Suslin theorem for $i=3$, the MerkurjevSuslin/Rost theorem for $i=4)$ : the map $H^{i}\left(F, \mu_{n}^{\otimes(i-1)}\right) \stackrel{\iota_{*}}{\longrightarrow} H^{i}(F, i-1)$ is injective, where $\iota^{\prime}: \mu_{n}^{\otimes(i-1)} \rightarrow \mathbf{Q} / \mathbf{Z}(i-1)$ is the natural inclusion. We need:

Lemma 7.2. Under the assumptions of proposition 7.1,

a) $\operatorname{Ker} \eta_{n}^{i}$ and $\operatorname{Ker} \eta_{m n}^{i}$ are killed by $\delta$.

b) $\operatorname{Ker} \eta_{n}^{i} \rightarrow \operatorname{Ker} \eta_{m n}^{i}$ is an isomorphism.

c) $\operatorname{Ker}\left(\right.$ Coker $\eta_{n}^{i} \rightarrow$ Coker $\left.\eta_{m n}^{i}\right)=\operatorname{Ker}\left(\right.$ Coker $\eta_{n}^{i} \rightarrow$ Coker $\left.\eta^{i}\right)$.

Proof. a) If $X(F) \neq \emptyset$, this follows from a classical specialization argument; in general, it follows from this case and a transfer argument.

b) follows from a).

c) We have a commutative diagram with exact rows:

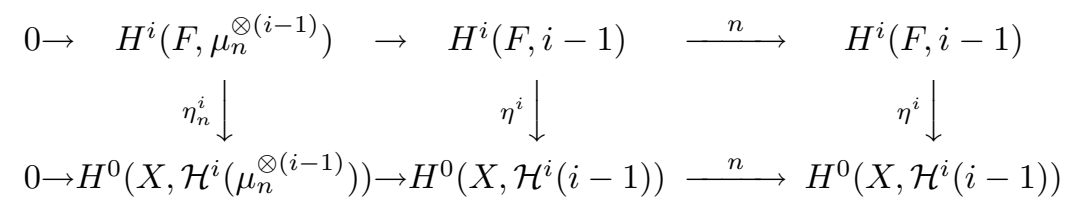

where the exactness of the bottom row follows from a diagram chase and the injectivity of $H^{i-1}\left(F(x), \mu_{n}^{\otimes(i-2)}\right) \rightarrow H^{i-1}(F(x), i-2)$ for all codimension 1 points of $X$. Let $x \in \operatorname{Ker}\left(\operatorname{Coker} \eta_{n}^{i} \rightarrow \operatorname{Coker} \eta^{i}\right), \tilde{x}$ a lift of $x$ in $H^{0}\left(X, \mathcal{H}^{i}\left(\mu_{n}^{\otimes(i-1)}\right)\right)$ and pick $\alpha \in H^{i}(F, i-1)$ such that $\eta^{i} \alpha=$ image of $\tilde{x}$. Since $n \alpha \in \operatorname{Ker} \eta^{i}$, we have $\delta n \alpha=0$ by a), hence $m n \alpha=0$. If $\beta$ is the corresponding element of $H^{i}\left(F, \mu_{m n}^{\otimes(i-1)}\right)$, it is clear that $\eta_{m n}^{i} \beta$ is the image of $\tilde{x}$ in $H^{0}\left(X, \mathcal{H}^{i}\left(\mu_{m n}^{\otimes(i-1)}\right)\right)$. 
Let $\iota: \mu_{n}^{\otimes(i-1)} \rightarrow \mu_{m n}^{\otimes(i-1)}$ (resp. $\pi: \mu_{m n}^{\otimes(i-1)} \rightarrow \mu_{m}^{\otimes(i-1)}$ ) denote the natural inclusion (resp. projection). Consider the commutative diagram with exact rows:

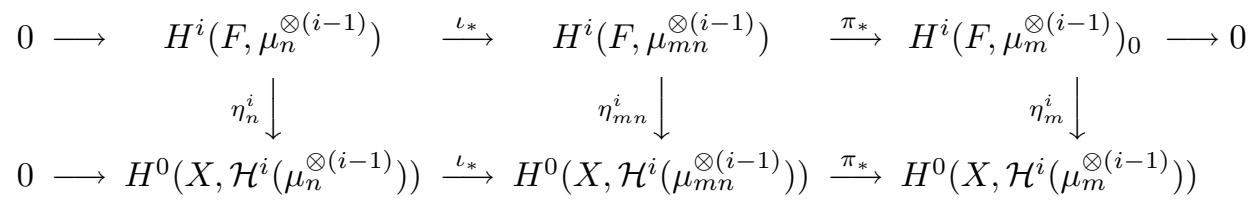

where $H^{i}\left(F, \mu_{m}^{\otimes(i-1)}\right)_{0}=\operatorname{Im} \pi_{*}$. From lemma $\left.7.2 \mathrm{~b}\right)$ and the snake lemma applied to this diagram, we derive an exact sequence

$$
0 \rightarrow \operatorname{Ker} \eta_{m}^{i} \cap \operatorname{Im}\left(H^{i}\left(F, \mu_{m n}^{\otimes(i-1)}\right) \stackrel{n}{\rightarrow} H^{i}\left(F, \mu_{m}^{\otimes(i-1)}\right)\right) \rightarrow \text { Coker } \eta_{n}^{i} \rightarrow \text { Coker } \eta_{m n}^{i}
$$

Proposition 7.1 now follows from lemma $7.2 \mathrm{c}$ ).

Proposition 7.3. Let $\operatorname{char} F \neq 2, i \geq 0$ and $\alpha \in H^{i} F$ be a decomposable element (i.e., a sum of symbols). Then $\alpha \in \operatorname{Im}\left(H^{i}\left(F, \mu_{4}^{\otimes(i-1)}\right) \stackrel{p}{\rightarrow} H^{i} F\right)$ if and only if $(-1) \cdot \alpha=0 \in H^{i+1} F$. Here $p$ is the map induced by the surjective homomorphism of Galois modules $\mu_{4}^{\otimes(i-1)} \rightarrow \mu_{2}^{\otimes(i-1)} \simeq \mathbf{Z} / 2$.

Proof. Let $\partial$ (resp. $\partial^{\prime}$ ) be the boundary map $H^{i} F \rightarrow H^{i+1} F$ corresponding to the short exact sequence $0 \rightarrow \mathbf{Z} / 2 \rightarrow \mu_{4}^{\otimes i} \rightarrow \mathbf{Z} / 2 \rightarrow 0\left(\right.$ resp. $0 \rightarrow \mathbf{Z} / 2 \rightarrow \mu_{4}^{\otimes(i-1)} \rightarrow$ $\mathbf{Z} / 2 \rightarrow 0)$. It follows from [18, lemma 1] that $\partial \alpha-\partial^{\prime} \alpha=(-1) \cdot \alpha$. If $\alpha$ is decomposable, then it obviously comes from $H^{i}\left(F, \mu_{4}^{\otimes i}\right)$ (reduce to a symbol $\left(a_{1}\right)$. $\ldots \cdot\left(a_{i}\right)$, where $a_{k} \in F^{*}$, and observe that each $\left(a_{k}\right)$ comes from the Kummer class of $a_{k}$ in $\left.H^{1}\left(F, \mu_{4}\right)\right)$, hence $\partial \alpha=0$ and the lemma follows.

Proposition 7.4. Suppose char $F \neq 2$ and $\delta=2$ in proposition 7.1 (e.g., $X$ is a quadric). For $i \leq 4$, the group

$$
\operatorname{Ker} \eta_{2}^{i} \cap \operatorname{Im}\left(H^{i}\left(F, \mu_{4}^{\otimes(i-1)}\right) \stackrel{2}{\rightarrow} H^{i} F\right)
$$

coincides with the group $\left(\operatorname{Ker} \eta_{2}^{i}\right)_{0}$ of the introduction. We have an exact sequence

$$
0 \rightarrow\left(\operatorname{Ker} \eta_{2}^{i}\right)_{0} \rightarrow \operatorname{Coker} \eta_{2}^{i} \rightarrow \operatorname{Coker} \eta^{i}
$$

Proof. Let $q$ be a quadratic form defining $X$ and $q^{\prime}=\langle a, b\rangle$ be any binary subform of $q$. Then $\operatorname{Ker} \eta_{2}^{i} \subseteq \operatorname{Ker}\left(H^{i} F \rightarrow H^{i} F(\sqrt{-a b})\right)=(-a b) \cdot H^{i-1} F$. Since $H^{i-1} F$ is generated by symbols $[31,32]$, we see that every element of $\operatorname{Ker} \eta_{2}^{i}$ is decomposable. Finally, the last claim follows from this and proposition 7.1.

Remark. The arguments in this section show in fact that proposition 7.4 follows for a given $i$ from the Milnor conjecture in degree $i$ (see the remark at the end of introduction).

\section{Constructing some maps}

In this section, we show how the Chow ring acts on étale and $\mathcal{H}$-cohomology with divisible coefficients. This action will be repeatedly used in the sequel.

Let $X$ be a smooth variety over $F, r \geq 1, i, j \geq 0$ and $k, k^{\prime} \in \mathbf{Z}$. We first recall that Galois cohomology of $F$ acts on the Hochschild-Serre and coniveau spectral 
sequences

$$
\begin{gathered}
H S_{2}^{p, q}\left(\mathbf{Z} / 2^{r}(k)\right):=H^{p}\left(F, H^{q}\left(\bar{X}, \mathbf{Z} / 2^{r}(k)\right)\right) \Rightarrow H^{p+q}\left(X_{\text {ét }}, \mathbf{Z} / 2^{r}(k)\right) \\
B O_{1}^{p, q}\left(\mathbf{Z} / 2^{r}(k)\right):=\coprod_{x \in X^{(p)}} H^{q-p}\left(F(x), \mathbf{Z} / 2^{r}(k-p)\right) \Rightarrow H^{p+q}\left(X_{\text {ét }}, \mathbf{Z} / 2^{r}(k)\right)
\end{gathered}
$$

in the following sense. There are pairings

$$
\begin{aligned}
& H^{n}\left(F, \mathbf{Z} / 2^{r}(j)\right) \otimes H S_{2}^{p, q}\left(\mathbf{Z} / 2^{r}(k)\right) \rightarrow H S_{2}^{p+n, q}\left(\mathbf{Z} / 2^{r}(j+k)\right) \\
& H^{n}\left(F, \mathbf{Z} / 2^{r}(j)\right) \otimes B O_{1}^{p, q}\left(\mathbf{Z} / 2^{r}(k)\right) \rightarrow B O_{1}^{p, q+n}\left(\mathbf{Z} / 2^{r}(j+k)\right)
\end{aligned}
$$

coming from the cup-product in Galois cohomology (and the restriction maps $H^{*}(F) \rightarrow H^{*}(F(x))$ for the coniveau spectral sequence). These pairings commute with differentials and induce compatible pairings

$$
\begin{aligned}
& H^{n}\left(F, \mathbf{Z} / 2^{r}(j)\right) \otimes H S_{s}^{p, q}\left(\mathbf{Z} / 2^{r}(k)\right) \rightarrow H S_{s}^{p+n, q}\left(\mathbf{Z} / 2^{r}(j+k)\right) \\
& H^{n}\left(F, \mathbf{Z} / 2^{r}(j)\right) \otimes B O_{s}^{p, q}\left(\mathbf{Z} / 2^{r}(k)\right) \rightarrow B O_{s}^{p, q+n}\left(\mathbf{Z} / 2^{r}(j+k)\right)
\end{aligned} \quad \text { for } s \leq \infty
$$

which are also compatible at the $E_{\infty}$-level with the pairings on the abutments

$$
H^{n}\left(F, \mathbf{Z} / 2^{r}(j)\right) \otimes H^{p+q}\left(X_{\text {ét }}, \mathbf{Z} / 2^{r}(k)\right) \rightarrow H^{p+q+n}\left(X_{\text {ét }}, \mathbf{Z} / 2^{r}(j+k)\right)
$$

(obtained from the maps $H^{i}\left(F, \mathbf{Z} / 2^{r}(k)\right) \rightarrow H^{i}\left(X, \mathbf{Z} / 2^{r}(k)\right)$ and cup-product in the étale cohomology of $X)$. This is classical for the Hochschild-Serre spectral sequence; for the coniveau spectral sequence, it is immediate from its exact couple construction out of étale cohomology with supports (this supersedes the sophisticated argument of [17, end of introduction]).

If $E / F$ is a finite extension, there are also compatible transfer maps.

We now play with these pairings a little bit. Using the cycle map

$$
C H^{j} X / 2^{r} \rightarrow H_{\text {ét }}^{2 j}\left(X, \mathbf{Z} / 2^{r}(j)\right)
$$

we get composite pairings

$$
\begin{aligned}
H^{i}\left(F, \mathbf{Z} / 2^{r}(k)\right) \otimes C H^{j} X \rightarrow H^{i}\left(F, \mathbf{Z} / 2^{r}(k)\right) \otimes H_{\text {ét }}^{2 j} & \left(X, \mathbf{Z} / 2^{r}(j)\right) \\
& \stackrel{\cup}{\longrightarrow} H_{\text {ét }}^{i+2 j}\left(X, \mathbf{Z} / 2^{r}(k+j)\right)
\end{aligned}
$$

hence, at the inductive limit:

$$
H^{i}(F, k) \otimes C H^{j} X \rightarrow H^{i+2 j}(X, k+j) .
$$

Recall now that $B O_{2}^{j, j}\left(\mathbf{Z} / 2^{r}(j)\right) \simeq C H^{j} X / 2^{r}$. We get therefore a pairing (similar to those in proposition 2.2):

$$
H^{i}\left(F, \mathbf{Z} / 2^{r}(k)\right) \otimes C H^{j} X \rightarrow H^{j}\left(X, \mathcal{H}^{i+j}\left(\mathbf{Z} / 2^{r}(k+j)\right)\right) .
$$

Passing to the inductive limit, we get pairings:

$$
H^{i}(F, k) \otimes C H^{j} X \rightarrow H^{j}\left(X, \mathcal{H}^{i+j}(k+j)\right) .
$$

It is not difficult to check that the maps of proposition 2.4 are special cases of (8.2).

We have:

Lemma 8.1. Let $\left(H^{*}(X, k+j)^{(m)}\right)_{m \geq 0}$ be the decreasing filtration on $H^{*}(X, k+j)$ defined by the coniveau spectral sequence. Then

a) The image of the map (8.1) is contained in $H^{i+2 j}(X, k+j)^{(j)}$.

b) The image of the map (8.2) consists of universal cycles. 
c) The diagram

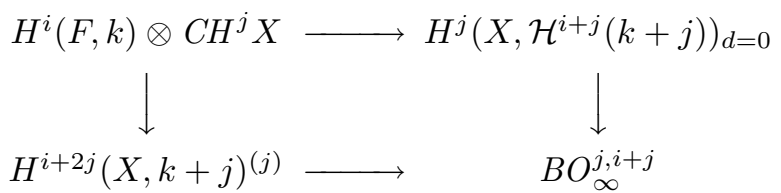

commutes, where the subscript $d=0$ denotes universal cycles and $B O_{\infty}^{j, i+j}$ denotes the $E_{\infty}$-term of the coniveau spectral sequence.

d) For $i=0$, 1, the triangle

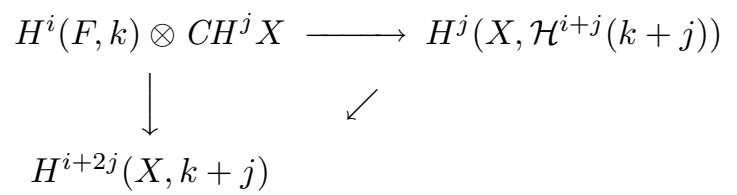

commutes, where the vertical map is (8.1), the horizontal map is (8.2) and the diagonal map is the edge homomorphism from the coniveau spectral sequence.

Recall that, in a spectral sequence $\left(E_{r}\right)$, an element $x \in E_{r}^{p, q}$ is called a universal cycle if $d_{s}^{p, q} x=0$ for all $s \geq r$ (this definition makes sense inductively).

Proof. It is enough to prove this for the maps defined at the finite coefficients level. By the multiplicativity of the coniveau spectral sequence, we are reduced to the case $i=k=0$. Then a), b), and c) follow from the fact that $B O_{1}^{p, q}=0$ for $p>q$. Finally, d) follows from c) and the definition of the edge homomorphism.

Let $A$ be a discrete topological $G_{F}$-module and $M$ an abelian group. There is a pairing

$$
H^{i}(F, A) \otimes M \rightarrow H^{i}(F, A \otimes M)
$$

for all $i \geq 0$, coming from the natural map $M \rightarrow \operatorname{Hom}_{G_{F}}(A, A \otimes M)$.

\section{Lemma 8.2.}

a) If $M$ is torsion-free, the above pairing is an isomorphism.

b) If $A$ is divisible, it is surjective.

c) If $A$ and $H^{i}(F, A)$ are divisible, it is bijective.

Proof. a) Reduce to the case where $M$ is finitely generated, hence free.

b) Reduce to the case where $M$ is torsion-free by using the commutative diagram:

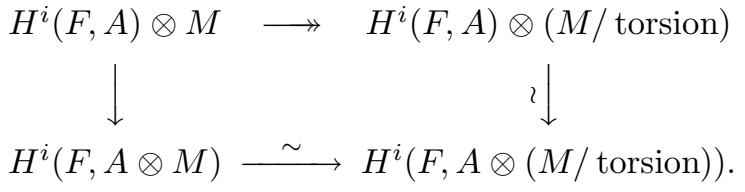

c) Use the same diagram, noting that now the top horizontal map is bijective as well.

Define a map

$$
\alpha=\alpha^{i, j, k}: H^{i}(F, k) \otimes C H^{j} X \rightarrow H^{i}\left(F, H^{2 j}(\bar{X}, j+k)\right)
$$


as the composite

$$
\begin{aligned}
H^{i}(F, k) \otimes C H^{j} X & \rightarrow H^{i}\left(F, C H^{j} X \otimes \mathbf{Q} / \mathbf{Z}(k)\right) \\
& \rightarrow H^{i}\left(F, C H^{j} \bar{X} \otimes \mathbf{Q} / \mathbf{Z}(k)\right) \stackrel{H^{i}\left(F, c l^{\prime}{ }^{j}(k)\right)}{\longrightarrow} H^{i}\left(F, H_{\text {ét }}^{2 j}(\bar{X}, j+k)\right) .
\end{aligned}
$$

We have:

Lemma 8.3. Let $\left(H^{*}(X, k+j)^{[m]}\right)_{m \geq 0}$ be the decreasing filtration on $H^{*}(X, k+j)$ defined by the Hochschild-Serre spectral sequence. Then

a) The image of the map (8.1) is contained in $H^{i+2 j}(X, k+j)^{[i]}$.

b) The image of $\alpha^{i, j, k}$ consists of universal cycles.

c) The diagram

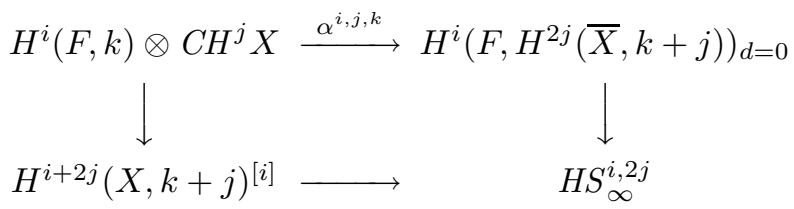

commutes, where the subscript $d=0$ denotes universal cycles and $H S_{\infty}^{i, 2 j}$ denotes the $E_{\infty}$-term of the Hochschild-Serre spectral sequence.

d) Suppose that $H^{2 j+1}(\bar{X}, k+j)=0$. Then, for $i=1$, the triangle

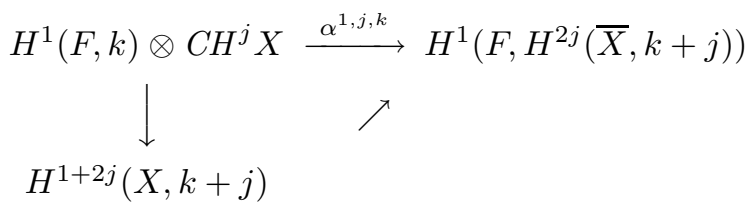

commutes, where the vertical map is (8.1) and the diagonal map is the edge homomorphism from the Hochschild-Serre spectral sequence.

Proof. We argue as above: it is enough to prove this for the maps defined at the finite coefficients level (we could have defined $\alpha$ as a limit, as before). By the multiplicativity of the Hochschild-Serre spectral sequence, we are reduced to the case $i=k=0$. Then a) is trivial, and b) and c) hold because $\alpha^{0, j, 0}$ factors through the ( $F$-rational) cycle map. Finally, d) follows as in the proof of lemma 8.1 from c) and the definition of the edge homomorphism.

The map $\alpha$ is the most important of the three maps introduced above in (8.1), (8.2), and (8.3). We are interested in its kernel and cokernel.

Lemma 8.4. If the geometric divisible cycle map is bijective, then

$\operatorname{Coker} \alpha^{i, j, k} \simeq \operatorname{Coker}\left(H^{i}\left(F, C H^{j} X \otimes \mathbf{Q} / \mathbf{Z}(k)\right) \rightarrow H^{i}\left(F, C H^{j} \bar{X} \otimes \mathbf{Q} / \mathbf{Z}(k)\right)\right)$.

If moreover $G_{F}$ acts trivially on $C H^{j} \bar{X}$, this cokernel is a quotient of

$$
\operatorname{Coker}\left(H^{i}(F, k) \otimes C H^{j} X \rightarrow H^{i}(F, k) \otimes C H^{j} \bar{X}\right) \text {. }
$$

Proof. The first claim follows from the definition of $\alpha^{i, j, k}$ and lemma 8.2. The second one follows from the commutative diagram

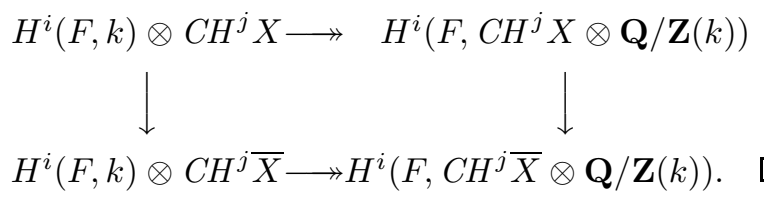


Lemma 8.5. Let $X$ be a quadric and $k=i \leq 3$. Then

a) If $\operatorname{dim} X \neq 2 j$ or $\operatorname{dim} X=2 j$ and $d(X)=1, \alpha^{i, j, i}$ is surjective.

b) Suppose $\operatorname{dim} X=2 j$ and $d(X)=d \neq 1$. Let $E=F(\sqrt{d})$. Then the composition

$H^{i}(E, i) \otimes C H^{j} X_{E} \stackrel{\alpha_{E}^{i, j, i}}{\longrightarrow} H^{i}\left(E, H^{2 j}(\bar{X}, i+j)\right) \stackrel{\operatorname{Cor}_{E / F}}{\longrightarrow} H^{i}\left(F, H^{2 j}(\bar{X}, i+j)\right)$ is surjective. For $i=0, \alpha^{0, j, 0}$ is itself surjective.

c) If $\operatorname{dim} X>2 j, \alpha^{i, j, i}$ is bijective.

Proof. In case a), the geometric cycle map is bijective by proposition 1.5 and $G_{F}$ acts trivially on $C H^{j} \bar{X}$ by proposition 1.1 , hence, by lemma 8.4 , it is enough to see that the map

$$
H^{i}(F, i) \otimes C H^{j} X \rightarrow H^{i}(F, i) \otimes C H^{j} \bar{X}
$$

is surjective. But, since $i \leq 3$, the group $H^{i}(F, i)$ is divisible by bijectivity of the 2-primary Galois symbol in this case. Surjectivity now follows from corollary 1.2, applied with $D=H^{i}(F, i)$.

In case b), $\alpha_{E}$ is surjective by a). It remains to prove that

$$
H^{i}\left(E, H^{2 j}(\bar{X}, i+j)\right) \stackrel{\operatorname{Cor}_{E / F}}{\longrightarrow} H^{i}\left(F, H^{2 j}(\bar{X}, i+j)\right)
$$

is surjective. By the geometric cycle map, the coefficients can be identified with $C H^{j} \bar{X} \otimes \mathbf{Q} / \mathbf{Z}(i) \simeq \operatorname{Ind}_{G_{E}}^{G_{F}} \mathbf{Q} / \mathbf{Z}(i)$ by corollary 1.3. Using Shapiro's lemma, the corestriction map can therefore be identified with

$$
H^{i}(E, i) \oplus H^{i}(E, i) \stackrel{\Sigma}{\longrightarrow} H^{i}(E, i)
$$

which is obviously surjective. Finally, the last claim is corollary 1.3.

In case c), the three maps out of which $\alpha$ is defined are bijective: the first one is by lemma $8.2 \mathrm{c}$ ), the second one by proposition $1.1 \mathrm{~b}$ ) and the last one by proposition 1.5 .

In section 11 we shall need the following lemma and its corollary for $(j, k)=$ $(2,1)$.

Lemma 8.6. Let $X$ be a quadric over F. Then:

a) If $F$ contains all 2-primary roots of unity, $\alpha^{0, j, k}$ is surjective for any $j, k$. The same holds if $k$ is even and $F(\sqrt{-1})$ contains all 2-primary roots of unity.

b) In general, Coker $\alpha^{0, j, k}$ is

(i) 0 if $\operatorname{dim} X>2 j$;

(ii) cyclic of order $\leq 2$ if $\operatorname{dim} X<2 j$;

(iii) cyclic of order $\leq 2^{t}$ if $\operatorname{dim} X=2 j$ and $d(X)=1$, where $t$ is the integer of proposition $1.1 \mathrm{~d}$ ).

Proof. By lemma 8.4,

Coker $\alpha^{0, j, k} \simeq \operatorname{Coker}\left(H^{0}\left(F, C H^{j} X \otimes \mathbf{Q} / \mathbf{Z}(k)\right) \rightarrow H^{0}\left(F, C H^{j} \bar{X} \otimes \mathbf{Q} / \mathbf{Z}(k)\right)\right)$.

Assume first that we are not in the case $\operatorname{dim} X=2 j$ and $d=d(X) \neq 1$. Applying proposition 1.1 and corollary 1.2 with $D=\mathbf{Q} / \mathbf{Z}(k)$, we see that the map on coefficients

$$
C H^{j} X \otimes \mathbf{Q} / \mathbf{Z}(k) \rightarrow C H^{j} \bar{X} \otimes \mathbf{Q} / \mathbf{Z}(k)
$$


can be identified with multiplication by $\left|\operatorname{Coker}\left(C H^{j} X \rightarrow C H^{j} \bar{X}\right)\right|$ on $\mathbf{Q} / \mathbf{Z}(k)$, except if $\operatorname{dim} X=2 j$ and $d(X)=1$, where it is $\operatorname{diag}\left(1,2^{t}\right)$ on $\mathbf{Q} / \mathbf{Z}(k) \oplus \mathbf{Q} / \mathbf{Z}(k)$ with $t$ as in prop. $1.1 \mathrm{~d}$ ). If $F$ contains all 2-primary roots of unity, this shows that $\alpha^{0, j, k}$ is surjective, and similarly if $k$ is even and $F(\sqrt{-1})$ contains all 2-primary roots of unity. For arbitrary $F$, if $\operatorname{dim} X>2 j$ then $C H^{j} X \rightarrow C H^{j} \bar{X}$ is surjective (prop. 1.1) hence $\alpha^{0, j, k}$ is an isomorphism. If $\operatorname{dim} X<2 j$ (resp. $\operatorname{dim} X=2 j$ and $d(X)=1)$, the map $C H^{j} X \rightarrow C H^{j} \bar{X}$ has cokernel cyclic of order $\leq 2\left(\right.$ resp. $\left.\leq 2^{t}\right)$, so that Coker $\alpha^{0, j, k}$ is cyclic of order $\leq 2\left(\right.$ resp. $\left.\leq 2^{t}\right)$.

Assume now that $\operatorname{dim} X=2 j$ and $d=d(X) \neq 1$; let $E=F(\sqrt{d})$. By corollary $1.3, \alpha^{0, j, k}$ can be identified with the map $H^{0}(F, k) \rightarrow H^{0}(E, k)$, so is surjective when $F$ and $E$ have the same number of 2-primary roots of unity, in particular if $F$ contains all 2-primary roots of unity; similarly if $k$ is even and $F(\sqrt{-1})$ contains all 2-primary roots of unity.

Corollary 8.7. Let $X$ be a quadric over $F$. Consider the composition

$$
\gamma: H^{j}\left(X, \mathcal{H}^{j}(j+k)\right) \rightarrow H^{2 j}(X, j+k) \rightarrow H^{0}\left(F, H^{2 j}(\bar{X}, j+k)\right)
$$

where the first (resp. second) map is an edge homomorphism of the coniveau (resp. Hochschild-Serre) spectral sequence. Then:

a) If $F$ contains all 2-primary roots of unity or if $k$ is even and $F(\sqrt{-1})$ contains all 2-primary roots of unity, $\gamma$ is surjective.

b) In general, Coker $\gamma$ is:

(i) 0 if $\operatorname{dim} X>2 j$;

(ii) cyclic of order $\leq 2$ if $\operatorname{dim} X<2 j$;

(iii) cyclic of order $\leq 2^{t}$ if $\operatorname{dim} X=2 j$ and $d(X)=1$, where $t$ is the integer of proposition $1.1 \mathrm{~d}$ ).

(iv) cyclic of order $\leq 2^{t_{E}}$ if $\operatorname{dim} X=2 j$ and $d(X)=d \neq 1$, where $t_{E}$ is the integer of proposition $1.1 \mathrm{~d}$ ) for $X_{E}$, with $E=F(\sqrt{d})$.

Proof. By lemmas 8.1 and 8.3, the composition

$$
H^{0}(F, k) \otimes C H^{j} X \rightarrow H^{j}\left(X, \mathcal{H}^{j}(j+k)\right) \stackrel{\gamma}{\rightarrow} H^{0}\left(F, H^{2 j}(\bar{X}, j+k)\right)
$$

coincides with $\alpha^{0, j, k}$. Therefore corollary 8.7 follows directly from lemma 8.6, except when $\operatorname{dim} X=2 j, d(X)=d \neq 1$ and $F$ does not contain enough roots of unity. But in this case, we have a commutative diagram:

$$
\begin{aligned}
& H^{0}(E, k) \otimes C H^{j} X_{E} \longrightarrow H^{j}\left(X_{E}, \mathcal{H}^{j}(j+k)\right) \stackrel{\gamma_{E}}{\longrightarrow} H^{0}\left(E, H^{2 j}(\bar{X}, j+k)\right) \\
& \operatorname{Cor}_{E / F} \downarrow \operatorname{Cor}_{E / F} \downarrow \\
& H^{0}(F, k) \otimes C H^{j} X \longrightarrow H^{j}\left(X, \mathcal{H}^{j}(j+k)\right) \stackrel{\gamma}{\longrightarrow} H^{0}\left(F, H^{2 j}(\bar{X}, j+k)\right)
\end{aligned}
$$

where the left Cor is best described from the $E_{1}$-term of the coniveau spectral sequence. By lemma 8.6, Coker $\gamma_{E}$ is cyclic of order $\leq 2^{t_{E}}$. But

$$
\operatorname{Cor}_{E / F}: H^{0}\left(E, H^{2 j}(\bar{X}, j+k)\right) \rightarrow H^{0}\left(F, H^{2 j}(\bar{X}, j+k)\right)
$$

is surjective: this follows from the bijectivity of the geometric cycle map and the fact that the Galois module $C H^{j} \bar{X}$ is isomorphic to $\operatorname{Ind}_{G_{E}}^{G_{F}} \mathbf{Z}$. This concludes the proof of corollary 8.7 . 


\section{The case $i=2$ : Proof of theorem 4}

Let $X$ be a smooth variety over $F$ such that $H^{1}(\bar{X}, \mathbf{Q} / \mathbf{Z})=0$. We have a cross of exact sequences

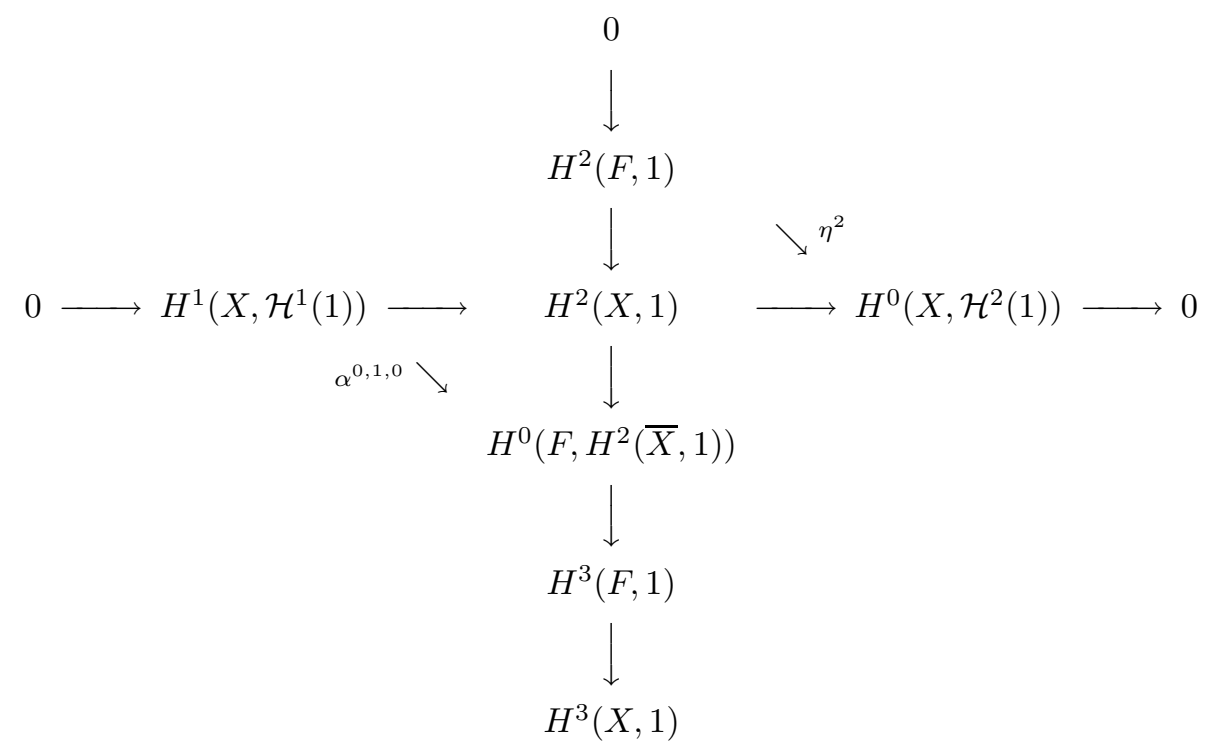

where the horizontal (resp. vertical) line is given by the coniveau (resp. HochschildSerre) spectral sequence with coefficients $\mathbf{Q} / \mathbf{Z}(1)$. The group $H^{1}\left(X, \mathcal{H}^{1}(1)\right)$ can be identified with $C H^{1} X \otimes \mathbf{Q} / \mathbf{Z}$ and $H^{1}\left(X, \mathcal{H}^{1}(1)\right) \rightarrow H^{2}(X, 1)$ with $c l^{\prime 1}$. The composite

$$
H^{1}\left(X, \mathcal{H}^{1}(1)\right) \rightarrow H^{2}(X, 1) \rightarrow H^{0}\left(F, H^{2}(\bar{X}, 1)\right)
$$

defined by the above cross can therefore be identified with the map $\alpha^{0,1,0}(8.3)$ of section 8 .

Proposition 9.1. For $X$ as above, there is an exact sequence

$$
0 \rightarrow \text { Coker } \eta^{2} \rightarrow \text { Coker } \alpha^{0,1,0} \rightarrow H^{3}(F, 1) \rightarrow H^{3}(X, 1) .
$$

Proof. This follows from the "lemma of the 700th" [33].

Corollary 9.2. For any (projective) quadric $X$ over $F, \eta^{2}$ is surjective.

Proof. $\alpha^{0,1,0}$ is surjective for all quadrics by lemma 8.5 .

\section{The case $i=3$ : Proof of theorem 5}

Let $X$ be a smooth variety over $F$ such that $H^{1}(\bar{X}, \mathbf{Q} / \mathbf{Z})=H^{3}(\bar{X}, \mathbf{Q} / \mathbf{Z})=0$. In the following cross of exact sequences the (broken) horizontal sequence comes from the coniveau spectral sequence and the vertical sequence from the Hochschild-Serre 
spectral sequence with coefficients $\mathbf{Q} / \mathbf{Z}(2)$.

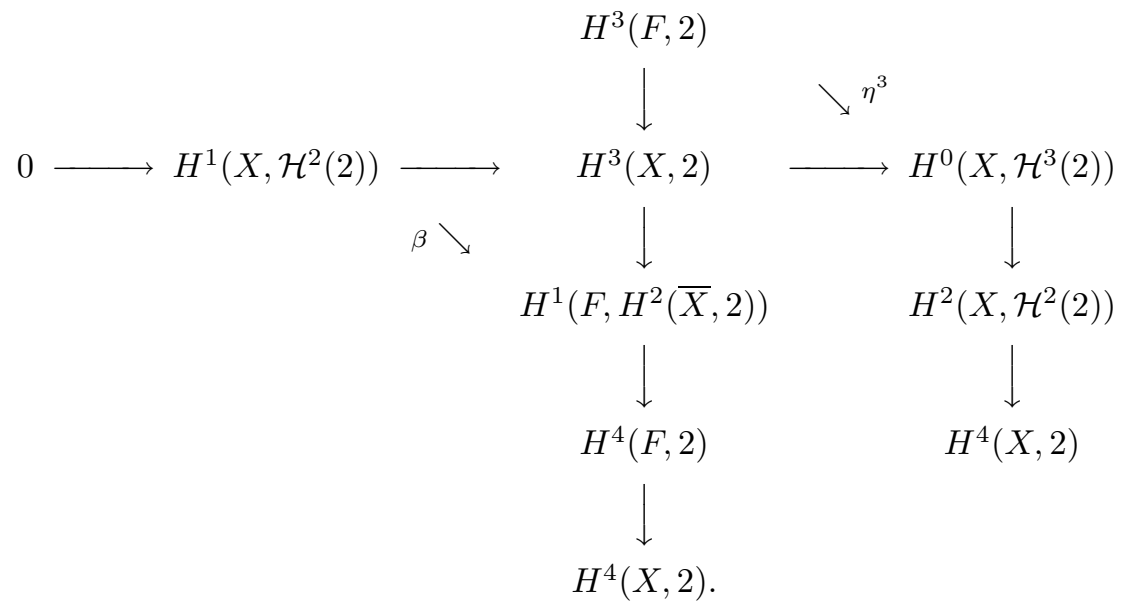

In this diagram, one can identify the group $H^{2}\left(X, \mathcal{H}^{2}(2)\right)$ with $C H^{2} X \otimes \mathbf{Q} / \mathbf{Z}$ and accordingly the map $H^{2}\left(X, \mathcal{H}^{2}(2)\right) \rightarrow H^{4}(X, 2)$ with $c l_{X}^{\prime 2}$. The above cross defines a composite homomorphism:

$$
\beta: H^{1}\left(X, \mathcal{H}^{2}(2)\right) \rightarrow H^{3}(X, 2) \rightarrow H^{1}\left(F, H^{2}(\bar{X}, 2)\right) .
$$

Proposition 10.1. For $X$ as above, there is a natural surjection

$$
\text { Coker } \eta^{3} \rightarrow \operatorname{Ker} c l_{X}^{\prime 2} \rightarrow 0 \text {. }
$$

Denote by $\left(\text { Coker } \eta^{3}\right)^{1}$ the kernel of this surjection. Then there is a natural exact sequence

$$
0 \rightarrow\left(\text { Coker } \eta^{3}\right)^{1} \rightarrow \text { Coker } \beta \rightarrow H^{4}(F, 2) \rightarrow H^{4}(X, 2) .
$$

Proof. The first claim is obvious and the second again follows from the lemma of the 700th [33].

Corollary 10.2. For any (projective) quadric $X$ over $F$, there is a natural isomorphism Coker $\eta^{3} \stackrel{\sim}{\longrightarrow} \operatorname{Ker} c l_{X}^{\prime 2}$.

Proof. We prove that $\beta$ is surjective for all quadrics. For this, we remark that the composition

$$
H^{1}(F, 1) \otimes C H^{1} X \rightarrow H^{1}\left(X, \mathcal{H}^{2}(2)\right) \stackrel{\beta}{\rightarrow} H^{1}\left(F, H^{2}(\bar{X}, 2)\right),
$$

where the first map is (8.2), coincides with $\alpha^{1,1,1}$ by lemmas 8.1 and 8.3. If $\operatorname{dim} X \neq$ 2 or $\operatorname{dim} X=2$ and $d(X)=1$, lemma 8.5 says that $\alpha^{1,1,1}$ is surjective, hence $\beta$ is surjective. If $\operatorname{dim} X=2$ and $d=d(X) \neq 1$, we use the same trick as in the proof of corollary 8.7: the composite $\operatorname{Cor}_{E / F} \circ \alpha_{E}^{1,1,1}$ is surjective, still by lemma 8.5, where $E=F(\sqrt{d})$. But there is a transfer map $H^{1}\left(X_{E}, \mathcal{H}^{2}(2)\right) \stackrel{\text { Cor }_{E / F}}{\longrightarrow} H^{1}\left(X, \mathcal{H}^{2}(2)\right)$ making the following diagram commute:

$$
\begin{array}{cccc}
H^{1}(E, 1) \otimes C H^{1} X_{E} \longrightarrow H^{1}\left(X_{E}, \mathcal{H}^{2}(2)\right) \stackrel{\beta_{E}}{\longrightarrow} H^{1}\left(E, H^{2}(\bar{X}, 2)\right) \\
\operatorname{Cor}_{E / F} \downarrow & & \operatorname{Cor}_{E / F} \downarrow \\
H^{1}(F, 1) \otimes C H^{1} X \longrightarrow & H^{1}\left(X, \mathcal{H}^{2}(2)\right) \stackrel{\beta}{\longrightarrow} H^{1}\left(F, H^{2}(\bar{X}, 2)\right),
\end{array}
$$

which concludes the proof in this case. 
Proposition 10.3 (cf. [17, th. 3]). For a quadric $X$ over a field of characteristic $\neq 2$, the cycle map

$$
c l_{X}^{2}: C H^{2} X \otimes \mathbf{Q} / \mathbf{Z} \rightarrow H^{4}(X, 2)
$$

is injective, except if $X$ is defined by an anisotropic Albert form. In this case, $\operatorname{Ker} c l_{X}^{2} \simeq \mathbf{Z} / 2$.

Proof. In view of corollary 10.2, we may assume $X$ anisotropic (prop. 2.5). If $\operatorname{dim} X>4$ or $\operatorname{dim} X=4$ and $d(X) \neq 1$, the map

$$
C H^{2} X \otimes \mathbf{Q} / \mathbf{Z} \rightarrow H^{0}\left(F, C H^{2} \bar{X} \otimes \mathbf{Q} / \mathbf{Z}\right)
$$

is an isomorphism by proposition 1.1 and the claim follows from the injectivity of the geometric cycle map (proposition 1.5). If $\operatorname{dim} X=1, C H^{2} X=0$ and the claim is trivial. If $\operatorname{dim} X=3$ and $Y$ is a hyperplane section of $X$, then the restriction map $C H^{2} X \otimes \mathbf{Q} / \mathbf{Z} \rightarrow C H^{2} Y \otimes \mathbf{Q} / \mathbf{Z}$ is an isomorphism by proposition 1.1 again, so that injectivity for $\operatorname{dim} X=2$ implies injectivity for $\operatorname{dim} X=3$. Let us assume $\operatorname{dim} X=2$. As in [17], we may assume $d(X)=1$ since, if $d=d(X) \neq 1$, the map $C H^{2} X \rightarrow C H^{2} X_{F(\sqrt{d})}$ is bijective (prop. 1.1 and 1.6). In this case, $C H^{2} X$ is generated by $h^{2}$, where $h \in C H^{1} X$ is the class of a hyperplane section (ibid.). Since $\operatorname{Ker} c l_{X}^{\prime 2}$ is killed by 2 (cor. 10.2 and prop. 2.5), it is enough to prove:

Lemma 10.4. If $X$ is an anisotropic quaternion quadric (= defined by a 2-fold Pfister form $)$, the image of $c l_{X}^{2}\left(h^{2}\right) \in H^{4} X$ in $H^{4}(X, 2)$ is $\neq 0$.

Proof. Indeed, $c l_{X}^{2}\left(h^{2}\right)=c \cdot c l_{X}^{1}(h)$ by [17, lemma 2], where $c$ is the Brauer class of the quadric, and $c l_{X}^{1}(h) \neq 0$ by [51, lemma $\left.\left.5.3 .2 \mathrm{c}\right)\right]$. Therefore $c l_{X}^{2}\left(h^{2}\right)$ maps to a nonzero element of $H^{2}\left(F, H^{2} \bar{X}\right) \simeq H^{2} F$. Its image in $H^{2}\left(F, H^{2}(\bar{X}, 2)\right) \simeq H^{2}(F, 1)$ is not 0 since the map $H^{2} F \rightarrow H^{2}(F, 1)$ is injective (compare beginning of proof of prop. 7.1). Therefore the image of $c l_{X}^{2}\left(h^{2}\right)$ in $H^{4}(X, 2)$ cannot be 0.

It remains to deal with the case of an anisotropic Albert quadric. In this case we have Ker $\eta_{2}^{3}=0$ [1, Satz 5.6] and Coker $\eta_{2}^{3} \simeq \mathbf{Z} / 2$ [17, theorem $2 \mathrm{~d}$ )], hence from proposition 7.4 and corollary 10.2 we get an injection

$$
\mathbf{Z} / 2 \simeq \operatorname{Coker} \eta_{2}^{3} \hookrightarrow \operatorname{Ker} c l_{X}^{\prime 2} .
$$

But propositions 1.5 and $1.1 \mathrm{~d}$ ) yield a commutative diagram with exact row

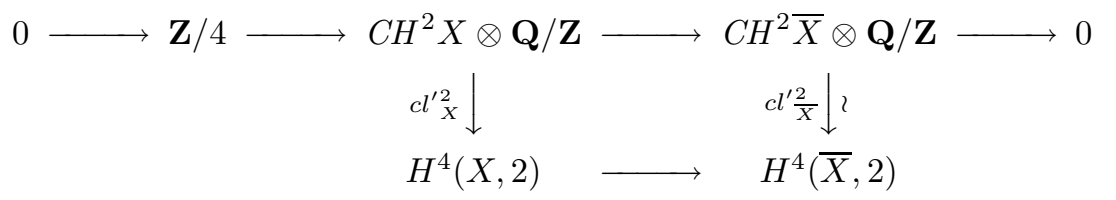

It follows that $\mathrm{Ker} \mathrm{Cl}_{X}^{\prime 2}$ is cyclic; as it is of exponent 2 by corollary 10.2 and proposition $2.5 \mathrm{~b}$ ), the map (10.1) is an isomorphism.

Theorem 5 follows from corollary 10.2 and proposition 10.3 . 


\section{The case $i=4$}

This case is similar to the former two, but more complicated.

For any smooth variety $X$ over $F$ define

$$
\bar{H}^{4}(X, 3)=\frac{H^{4}(X, 3)}{H^{4}(F, 3)+H^{2}\left(X, \mathcal{H}^{2}(3)\right)+\bigoplus_{[E: F]<+\infty} H^{2}(E, 2) \otimes C H^{1} X_{E}},
$$

where $H^{2}\left(X, \mathcal{H}^{2}(3)\right) \rightarrow H^{4}(X, 3)$ is the edge homomorphism of the coniveau spectral sequence and the map $\bigoplus_{[E: F]<+\infty} H^{2}(E, 2) \otimes C H^{1} X_{E} \rightarrow H^{4}(X, 3)$ comes from (8.1) via transfer.

Suppose that $H^{1}(\bar{X}, \mathbf{Q} / \mathbf{Z})=H^{3}(\bar{X}, \mathbf{Q} / \mathbf{Z})=0$. We then have two exact sequences

$$
\begin{aligned}
0 \rightarrow H^{1}\left(X, \mathcal{H}^{3}(3)\right) \rightarrow \frac{H^{4}(X, 3)}{H^{2}\left(X, \mathcal{H}^{2}(3)\right)} \rightarrow & H^{0}\left(X, \mathcal{H}^{4}(3)\right) \longrightarrow \\
& \stackrel{d_{2}^{0,4}}{\longrightarrow} H^{2}\left(X, \mathcal{H}^{3}(3)\right) \rightarrow H^{5}(X, 3)
\end{aligned}
$$

(coniveau)

$$
\begin{aligned}
0 \rightarrow \operatorname{Ker}\left(H^{2}\left(F, H^{2}(\bar{X}, 3)\right) \stackrel{d_{3}^{2,2}}{\longrightarrow} H^{5}(F, 3)\right) & \rightarrow \\
& \rightarrow \frac{H^{4}(X, 3)}{H^{4}(F, 3)} \rightarrow H^{0}\left(F, H^{4}(\bar{X}, 3)\right)
\end{aligned}
$$

(Hochschild-Serre).

The exact sequence (11.2) identifies $\operatorname{Ker} d_{3}^{2,2}$ to a subgroup of $\frac{H^{4}(X, 3)}{H^{4}(F, 3)}$. For the purpose of stating proposition 11.1 below, let $H^{2}\left(X, \mathcal{H}^{2}(3)\right)_{0}$ denote its intersection with $\operatorname{Im}\left(H^{2}\left(X, \mathcal{H}^{2}(3)\right) \rightarrow H^{4}(X, 3) \rightarrow \frac{H^{4}(X, 3)}{H^{4}(F, 3)}\right)$ (the first map in this composition being an edge homomorphism of the coniveau spectral sequence).

Proposition 11.1. For $X$ as above, there are exact sequences

$$
\bar{H}^{4}(X, 3) \rightarrow \text { Coker } \eta^{4} \rightarrow H^{2}\left(X, \mathcal{H}^{3}(3)\right) \rightarrow H^{5}(X, 3)
$$

and

$$
\frac{\operatorname{Ker}\left(H^{2}\left(F, H^{2}(\bar{X}, 3)\right) \stackrel{d_{3}^{2,2}}{\longrightarrow} H^{5}(F, 3)\right)}{H^{2}\left(X, \mathcal{H}^{2}(3)\right)_{0}+\bigoplus_{[E: F]<+\infty} H^{2}(E, 2) \otimes C H^{1} X_{E}} \rightarrow \bar{H}^{4}(X, 3) \rightarrow \frac{H^{0}\left(F, H^{4}(\bar{X}, 3)\right)}{H^{2}\left(X, \mathcal{H}^{2}(3)\right)}
$$

where the factor

$$
\bigoplus_{[E: F]<+\infty} H^{2}(E, 2) \otimes C H^{1} X_{E}
$$

comes from

$$
\sum_{[E: F]<+\infty} \operatorname{Cor}_{E / F} \circ \alpha_{E}^{2,1,2}
$$

Proof. This follows from the exact sequences (11.1) and (11.2), using lemma 8.3. 
Consider the cross of exact sequences

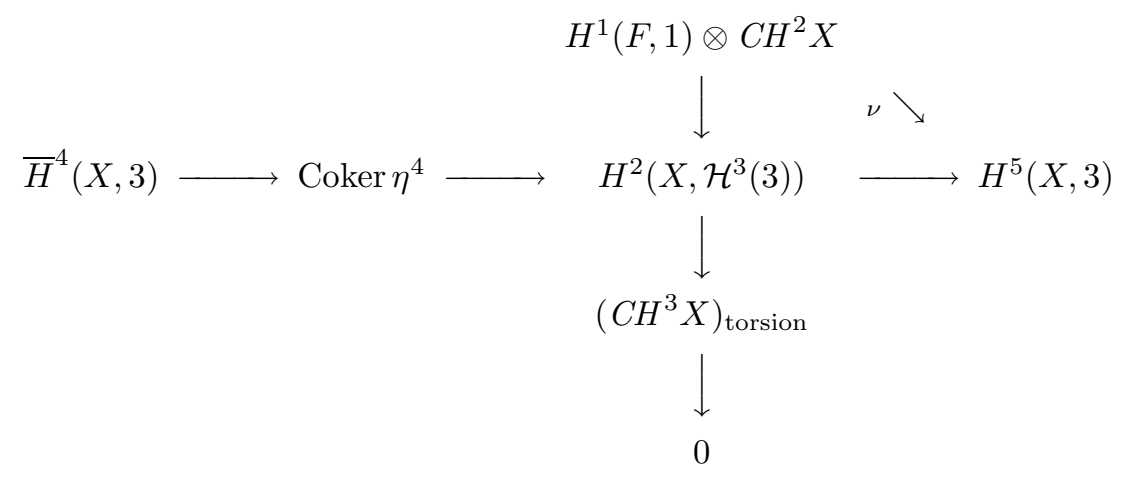

coming from propositions 2.4 (for $i=3$ ) and 11.1. From diagram (11.3) and the lemma of the 700th, we deduce a complex

$$
\bar{H}^{4}(X, 3) \rightarrow \text { Coker } \eta^{4} \rightarrow\left(C H^{3} X\right)_{\text {torsion }} \rightarrow \text { Coker } \nu
$$

which is exact at $\left(\mathrm{CH}^{3} \mathrm{X}\right)_{\text {torsion }}$ and whose homology at Coker $\eta^{4}$ is a quotient of $\operatorname{Ker} \nu$.

Lemma 11.2. Let $X$ be a quadric. Then $\bar{H}^{4}(X, 3)$ is cyclic of order $\leq 4$; it is 0 if $\operatorname{dim} X=1, \operatorname{dim} X>4$ or if $F$ contains all 2-primary roots of unity.

Proof. By lemma 8.5, $\alpha^{2,1,2}$ is surjective, except if $\operatorname{dim} X=2$ and $d=d(X) \neq 1$; in this case, $\operatorname{Cor}_{E / F} \circ \alpha_{E}^{2,1,2}$ is surjective. It follows that for any quadric, the group on the left of $\bar{H}^{4}(X, 3)$ in the second exact sequence of proposition 11.1 is 0 . Therefore $\bar{H}^{4}(X, 3)$ injects into $\frac{H^{0}\left(F, H^{4}(\bar{X}, 3)\right)}{H^{2}\left(X, \mathcal{H}^{2}(3)\right)}$. But the latter group is cyclic of order as in the statement of lemma 11.2 , by corollary 8.7 and proposition 1.1 .

Lemma 11.3. If $\operatorname{dim} X=1$ or $\operatorname{dim} X>4$, the map $\nu$ of diagram (11.3) is injective. If $\operatorname{dim} X>4$, there is a canonical isomorphism

$$
\text { Coker } \nu \simeq H^{5}(F, 3) \oplus\left(H^{3}(F, 2) \otimes C H^{1} X\right) .
$$

Proof. The case $\operatorname{dim} X=1$ is obvious, since then $C H^{2} X=0$. Suppose $\operatorname{dim} X>4$. By lemma $8.1 \mathrm{~d}$ ), the map $\nu$ coincides with (8.1). By lemma $8.5 \mathrm{c}), \alpha^{1,2,1}$ is bijective. Therefore, by lemma $8.3 \mathrm{~d}$ ), $\nu$ is (split-) injective. This argument shows that, in the Hochschild-Serre spectral sequence for coefficients $\mathbf{Q} / \mathbf{Z}(3)$, the term $E_{2}^{1,4}$ is isomorphic to $H^{1}(F, 1) \otimes C H^{2} X$ and consists of universal cycles. The same holds for all the terms $E_{2}^{p, 2} \simeq H^{p}(F, 2) \otimes C H^{1} X$ by the same argument, since $C H^{1} X \stackrel{\sim}{\longrightarrow} C H^{1} \bar{X}$ for $\operatorname{dim} X \geq 3$. Finally the term $E_{2}^{0,4}$ also consists of universal cycles by lemma 11.2. Hence we get a canonical isomorphism:

$$
H^{5}(X, 3) \simeq H^{5}(F, 3) \oplus\left(H^{3}(F, 2) \otimes C H^{1} X\right) \oplus\left(H^{1}(F, 1) \otimes C H^{2} X\right)
$$

and the second part of the lemma follows. 


\section{Proof of theorems 6 and 7}

Proof of theorem 6. Claim (1) follows from (11.4), lemma 11.2 and lemma 11.3. We now check (2) and (3). Let $q$ be a quadratic form defining the quadric $X$.

a) If $\operatorname{dim} q=3$, Coker $\eta^{4}=0$ by (11.4), lemma 11.2 and lemma 11.3.

b) If $\operatorname{dim} q>6$, part (1) of theorem 6 implies that Coker $\eta^{4}$ injects into the group $\left(C H^{3} X\right)_{\text {torsion, }}$, hence has order $\leq 2$ by Karpenko's results (proposition 1.6). If $\operatorname{dim} q>12,\left(\mathrm{CH}^{3} X\right)_{\text {torsion }}=0$ by the same results, and so Coker $\eta^{4}=0$.

c) If $\operatorname{dim} q=8, d_{ \pm} q=1$, and $q$ is not similar to a Pfister form, then $C H^{3} X$ is torsion-free by [20, th. 8.3] and Coker $\eta^{4}=0$ by (1).

The case of a 2-fold Pfister neighbour and that of an $i$-fold Pfister neighbour for $i \geq 4$ is covered by a) and b) respectively.

It remains to prove claim (3) in the case of a 3 -fold neighbour. We may assume the quadric to be of dimension at least 5 by proposition $2.5 \mathrm{c}$ ). Then the claim follows from:

Lemma 12.1. Suppose $\operatorname{dim} X>4$ and $X$ is defined by a neighbour of a 3-fold Pfister form. Then the map of theorem 6 (1)

$$
\left(C H^{3} X\right)_{\text {torsion }} \rightarrow H^{5}(F, 3) \oplus\left(H^{3}(F, 2) \otimes C H^{1} X\right)
$$

is injective.

Proof. By the same argument as in the proof of lemma 11.3, we get a canonical isomorphism

$$
H^{3}(X, 2) \simeq H^{3}(F, 2) \oplus\left(H^{1}(F, 1) \otimes C H^{1} X\right)
$$

compatible with the maps of section 8. The diagram analogous to (11.3)

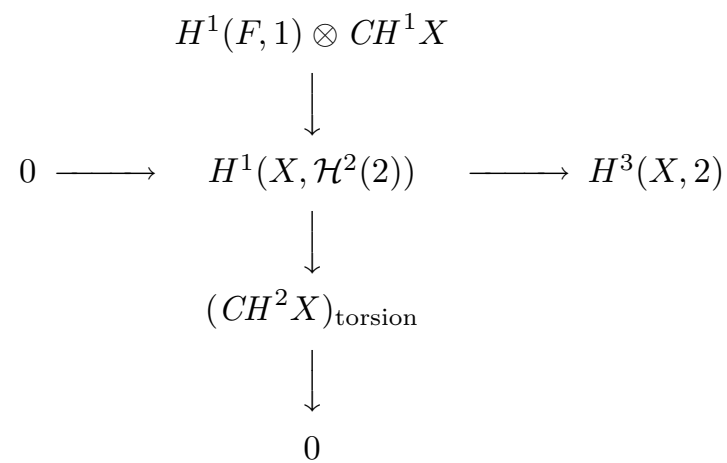

yields an injection

$$
\left(C H^{2} X\right)_{\text {torsion }} \longleftrightarrow H^{3}(F, 2) .
$$

Tensoring this injection by $C H^{1} X$ yields another injection (since $C H^{1} X$ is torsion-free)

$$
\left(C H^{2} X\right)_{\text {torsion }} \otimes C H^{1} X \longleftrightarrow H^{3}(F, 2) \otimes C H^{1} X .
$$

The way these maps are defined shows that the diagram

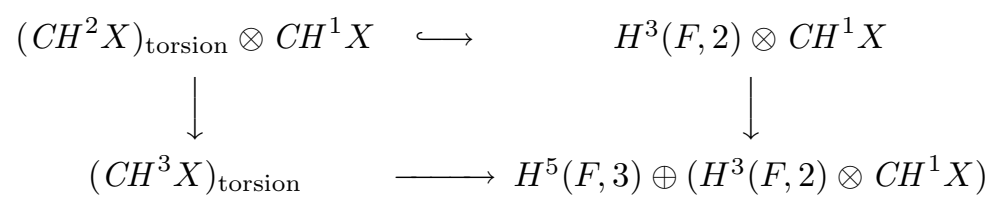


commutes, where the left vertical map is multiplication, the right vertical map is inclusion, the top horizontal map has just been defined and the bottom horizontal map comes from theorem 6 (1). Since $C H^{1} X \simeq \mathbf{Z}$ and $\left(C H^{2} X\right)_{\text {torsion }} \simeq \mathbf{Z} / 2$ by propositions 1.1 and 1.6, this shows that the latter map is nonzero. Since, by proposition 1.6, $\left(\mathrm{CH}^{3} \mathrm{X}\right)_{\text {torsion }}$ has order at most 2, this map is injective, which is what we wanted (moreover, we get that $C H^{1} \mathrm{X} \otimes\left(C H^{2} X\right)_{\text {torsion }} \rightarrow\left(C H^{3} X\right)_{\text {torsion }}$ is bijective, which fits with what Karpenko's method gives [21]).

Proof of theorem 7. In the case where $X$ is defined by an anisotropic Albert form $q$, we define a homomorphism

$$
\rho: H^{1} F \rightarrow \text { Coker } \eta_{2}^{4}
$$

by cup-product with a representative of Coker $\eta_{2}^{3} \simeq \mathbf{Z} / 2$.

Recall [17] that such a representative $e$ is constructed as follows: let $K=F(X)$ and $q_{K} \sim a \tau$, where $a \in K^{*}$ and $\tau$ is a 2-fold Pfister form. Then $e=(a) \cdot e^{2}(\tau)$.

The composition $H^{1} F \rightarrow$ Coker $\eta_{2}^{4} \rightarrow$ Coker $\eta^{4}$ has infinite image if we pass from $F$ to the rational function field $F(t)$. Indeed, $F$ is infinite since $q$ is anisotropic. Then, for any $\alpha \in F$, the element

$$
(t-\alpha) \cdot e \in H^{4} F(X)(t) \hookrightarrow H^{4}(F(X)(t), 3)
$$

is unramified over $F(t)$, and its residue at $\alpha$ is $e \in H^{3}(F(X), 2)$. This shows that the classes of the $(t-\alpha) \cdot e$ in Coker $\eta_{F(t)}^{4}$ are linearly independent.

In the case of a virtual Albert form $q$ of discriminant $d$, we define a homomorphism

$$
\rho^{\prime}: E^{*} \rightarrow \text { Coker } \eta_{2}^{4}
$$

simply as the composite

$$
E^{*} \stackrel{\rho}{\longrightarrow} \text { Coker } \eta_{2, q_{E}}^{4} \stackrel{\text { Cor }_{E / F}}{\longrightarrow} \text { Coker } \eta_{2}^{4}
$$

where Coker $\eta_{2, q_{E}}^{4}$ is the cokernel attached to the form $q_{E}(E=F(\sqrt{d}))$. We show just as above that Coker $\eta_{F(t)}^{4}$ is infinite, now using rational points of $\mathbf{A}_{E}^{1}$ not defined over $F$.

These arguments can be summarized by noting that the groups Coker $\eta^{i}$ are cycle modules in the sense of [42] and using the exact sequence

$$
0 \rightarrow \operatorname{Coker} \eta_{F}^{4} \rightarrow \operatorname{Coker} \eta_{F(t)}^{4} \rightarrow \bigoplus_{y \in \mathbf{A}_{(0)}^{1}} \operatorname{Coker} \eta_{F_{y}}^{3} \rightarrow 0
$$

$c f$. the exact sequence $(*)$ in the introduction.

Exercise. Let $X$ be an anisotropic quadric, $a \in F^{*}$ and $E=F(\sqrt{a})$ a quadratic extension such that $X_{E}$ is isotropic.

a) Show that extension of scalars defines a homomorphism

$$
\theta_{E}^{n}: H^{0}\left(X, \mathcal{H}^{n}\right) \rightarrow H^{n} E
$$

for all $n \geq 0$.

b) Define a 0 -sequence

$$
H^{3} F \stackrel{\cdot(a)}{\longrightarrow} \operatorname{Ker} \theta_{E}^{4} \rightarrow \operatorname{Ker}\left(C H^{3} X \rightarrow C H^{3} X_{E}\right)
$$

(proceed by a diagram chase, as in [27] for the definition of the map Ker $\eta_{2}^{4} \rightarrow$ $\left.\operatorname{Ker}\left(H^{2}\left(X, \mathcal{K}_{3}\right) \rightarrow H^{2}\left(X_{E}, \mathcal{K}_{3}\right)\right)\right)$ 
c) Compare the map $\operatorname{Ker} \theta_{E}^{4} \rightarrow \operatorname{Ker}\left(C H^{3} X \rightarrow C H^{3} X_{E}\right)$ of b) with the map Coker $\eta_{2}^{4} \rightarrow\left(C H^{3} X\right)_{\text {torsion }}$ deduced from theorem 6 .

\section{Appendix A. Quadrics of dimension 0 and 1}

Proposition A.1. Let $\operatorname{dim} X=1$ and suppose the Milnor conjecture holds in degree $i-2$. Then Coker $\eta^{i}=0$.

Proof. It follows from the cross of exact sequences

$$
\begin{aligned}
& H^{i}(F, i-1) \\
& 0 \rightarrow H^{1}\left(X, \mathcal{H}^{i-1}(i-1)\right) \rightarrow \quad H^{i}(X, i-1) \quad \rightarrow H^{0}\left(X, \mathcal{H}^{i}(i-1)\right) \rightarrow 0 \\
& H^{i-2}\left(F, H^{2}(\bar{X}, i-2)\right)
\end{aligned}
$$

(coniveau and Hochschild-Serre), by using the fact that the composition

$$
H^{i-2}(F, i-2) \otimes C H^{1} X \rightarrow H^{1}\left(X, \mathcal{H}^{i-1}(i-1)\right) \rightarrow H^{i-2}\left(F, H^{2}(\bar{X}, i-2)\right)
$$

is surjective. As above, this is deduced from the divisibility of $H^{i-2}(F, i-2)$ and corollary 1.2 .

In the following we present analogues of proposition A.1 and proposition 3 for 0 -dimensional quadrics. Let $L=F(\sqrt{d})$ be a quadratic extension with Galois group $G=\operatorname{Gal}(L / F)$.

Proposition A.2. Suppose the Milnor conjecture holds in degree $i-1$. Then

a) The restriction map

$$
H^{i}(F, i-1) \rightarrow\left(H^{i}(L, i-1)\right)^{G}
$$

is surjective.

b) The restriction map

$$
H^{i} F \rightarrow\left(H^{i} L\right)^{G}
$$

has kernel $(d) \cdot H^{i-1} F$ and cokernel isomorphic to

$$
\left\{x \in(d) \cdot H^{i-1} F \mid(-1) \cdot x=0\right\} .
$$

Proof. a) Let $\sigma$ be the generator of $G$ and consider the exact sequence of Galoismodules

$$
0 \rightarrow \mathbf{Z} \rightarrow \mathbf{Z}[G] \stackrel{1-\sigma}{\longrightarrow} \mathbf{Z}[G] \rightarrow \mathbf{Z} \rightarrow 0
$$

Tensoring with $\mathbf{Q} / \mathbf{Z}(i-1)$ gives a spectral sequence converging to 0 , with $E_{1}$ term:

$$
\begin{array}{rlllll}
0 \longrightarrow H^{0}(F, i-1) & \stackrel{\text { Res }}{\longrightarrow} H^{0}(L, i-1) & \stackrel{1-\sigma}{\longrightarrow} H^{0}(L, i-1) & \stackrel{\text { Cor }}{\longrightarrow} H^{0}(F, i-1) & \longrightarrow 0 \\
& \cdots
\end{array}
$$


Here the $d^{2}$-differentials go 2 steps right and 1 step up. The norm map

$$
H^{i-1}(L, i-1) \stackrel{\text { Cor }}{\longrightarrow} H^{i-1}(F, i-1)
$$

is computed as the norm map

$$
K_{i-1}^{\mathrm{M}} L \otimes \mathbf{Q} / \mathbf{Z} \stackrel{N}{\longrightarrow} K_{i-1}^{\mathrm{M}} F \otimes \mathbf{Q} / \mathbf{Z},
$$

which is obviously surjective. Hence

$$
H^{i}(F, i-1) \stackrel{\text { Res }}{\longrightarrow} H^{i}(L, i-1) \stackrel{1-\sigma}{\longrightarrow} H^{i}(L, i-1)
$$

is exact, i.e., $\eta^{i}$ is surjective.

This discussion holds for any cyclic extension $L / F$ with the degree $[L: F]$ prime to char $F$.

b) The statement on the kernel is classical [1]; the statement on the cokernel follows from this one and a), by using proposition 7.4, which is shown to apply in this case just as in section 7 .

\section{Appendix B. Application to real quadrics}

In this section, we examine the case when the ground field is the field $\mathbf{R}$ of real numbers. The real anisotropic quadric of dimension $d$, denoted $Q_{d}$, is defined by the $(d+2)$-dimensional form $(d+2)\langle 1\rangle$. As all anisotropic quadratic forms over $\mathbf{R}$ are Pfister neighbours, the anisotropic quadrics can be grouped according to the level of their function fields ( $c f .[10, \S 3])$. As observed in [10, §3], the unramified cohomology groups are invariant for the set of quadrics grouped in this manner. In [10], it was proved that the unramified cohomology group $H^{0}\left(Q_{d}, \mathcal{H}^{3}\right)$ is 0 for $d \leq 6$ and that the map $\mathbf{Z} / 2 \simeq H^{3}(\mathbf{R}) \rightarrow H^{0}\left(Q_{d}, \mathcal{H}^{3}\right)$ is bijective for $d>7$. This result was used to show that the cycle map in codimension two

$$
C H^{2} Q_{d} / 2 \rightarrow H^{4} Q_{d}
$$

is injective for all quadrics $Q_{d}$ (compare also [17, th. 3]).

We extend these results in this section, and use them to get extra information on the cohomology of real quadrics. Concerning unramified cohomology, results can be expressed concisely in a striking way:

Theorem B.1. Let $F=\mathbf{R}$ and $i \leq 4$. Then, for any $d>0$, the map $H^{i}(\mathbf{R}) \rightarrow$ $H^{0}\left(Q_{d}, \mathcal{H}^{i}\right)$ is surjective. If $d>2^{i}-2$ it is bijective; if $d \leq 2^{i}-2, H^{0}\left(Q_{d}, \mathcal{H}^{i}\right)=0$.

Proof. For $i=2$ it is classical and for $i=3$ this is the main result of [10]. Note however that these cases also follow directly from proposition 7.4, theorem 6 and the fact that there are no anisotropic Albert quadrics over $\mathbf{R}$ in view of

Lemma B.2. With notation as above, if $F=\mathbf{R}$ then $\left(\operatorname{Ker} \eta_{2}^{i}\right)_{0}=0$ for all quadrics $X$ and all $i \geq 1$.

Proof. Cup-product by $(-1)$ is injective on $H^{*} \mathbf{R}$.

Let us now prove theorem B.1 in the case $i=4$. For $d>14$ and for $6<d \leq 14$, this follows from theorem 3.4 and corollary 8 . For $3<d \leq 6$, we may assume $d=3$; then $c d\left(\mathbf{R}\left(Q_{3}\right)\right)=3[47]$, hence $H^{4}\left(\mathbf{R}\left(Q_{3}\right)\right)=0$ and a fortiori $H^{0}\left(Q_{3}, \mathcal{H}^{4}\right)=0$. The argument is the same for $d \leq 2$. 
Theorem B.1 implies in particular that, in the coniveau spectral sequence

$$
E_{2}^{p, q}=H^{p}\left(Q_{d}, \mathcal{H}^{q}\right) \Rightarrow H^{p+q} Q_{d},
$$

$E_{2}^{0, q}$ consists of universal cycles for $q \leq 4$. This in turn implies:

\section{Theorem B.3.}

a) For any $d \geq 1$, we have:

$$
\operatorname{dim} H^{1}\left(Q_{d}, \mathcal{H}^{2}\right)+\operatorname{dim} H^{0}\left(Q_{d}, \mathcal{H}^{3}\right)=\operatorname{dim} H^{3} Q_{d} ;
$$

$\operatorname{dim} C H^{2} Q_{d} \otimes \mathbf{Z} / 2+\operatorname{dim} H^{1}\left(Q_{d}, \mathcal{H}^{3}\right)+\operatorname{dim} H^{0}\left(Q_{d}, \mathcal{H}^{4}\right)=\operatorname{dim} H^{4} Q_{d}$.

b) The "edge homomorphism" $H^{2}\left(Q_{d}, \mathcal{H}^{3}\right) \rightarrow H^{5} Q_{d}$ is injective.

Corollary B.4. We have:

$$
\operatorname{dim} H^{1}\left(Q_{d}, \mathcal{H}^{2}\right)= \begin{cases}0 & \text { for } d=1 \\ 1 & \text { for } d=2 \\ 2 & \text { for } 3 \leq d \leq 6 \\ 1 & \text { for } d>6\end{cases}
$$

and

$$
\operatorname{dim} H^{1}\left(Q_{d}, \mathcal{H}^{3}\right)= \begin{cases}0 & \text { for } d \leq 3 \\ 1 & \text { for } 4 \leq d \leq 6 \\ 2 & \text { for } 7 \leq d \leq 14 \\ 1 & \text { for } d>14\end{cases}
$$

Sketch of proof. An inductive argument allows one to compute $\operatorname{dim} H^{*} Q_{d}$. The method is to first compute the cohomology of the corresponding affine quadric by reduction to the complex case. The answer is [19]:

$$
\operatorname{dim} H^{n} Q_{d}= \begin{cases}{\left[\frac{n}{2}\right]+1} & \text { if } d \geq n \\ {\left[\frac{2 d-n}{2}\right]+1} & \text { if } d \leq n .\end{cases}
$$

The corollary follows from this fact, from theorem B.1, theorem B.3, and from (for the value of $\left.\operatorname{dim} C H^{2} Q_{d} \otimes \mathbf{Z} / 2\right)$ propositions 1.1 and 1.6.

Further results on $H^{2}\left(Q_{d}, \mathcal{H}^{3}\right), H^{1}\left(Q_{d}, \mathcal{H}^{4}\right)$ and the cycle map $c l_{Q_{d}}^{3}$ can be obtained at a higher cost [19].

\section{Appendix C. The unramified Witt ring}

In this appendix, we prove theorem 9 , which we now recall:

Theorem C.1. Let $i \leq 4$. For any quadric $X$, the composition

$$
\text { Coker } \chi^{i} \rightarrow \text { Coker } \eta_{2}^{i} \rightarrow \text { Coker } \eta^{i}
$$

is injective, where

$$
\chi^{i}: I^{i} F / I^{i+1} F \rightarrow I_{n r}^{i}(F(X) / F) / I_{n r}^{i+1}(F(X) / F)
$$

is the map induced by extension of scalars and the first map in the composition is induced by the cohomological invariant $\bar{e}^{i}$. 
Lemma C.2. Let $C$ be a conic over $F$ and $K=F(C)$. Let $c \in H^{2} F$ be the class of the quaternion algebra corresponding to $C$. Then, for $i \leq 4$,

a) $\operatorname{Ker} \eta_{2}^{i}=c \cdot H^{i-2} F$.

b) The natural map

$$
I^{i+1} F \rightarrow I_{n r}^{i+1}(K / F)
$$

is surjective.

Proof. a) has been given earlier in the paper for $i \leq 3$ and is proven in [30, prop. 3.15] for $i=4$. Let us prove b). By a result of Parimala [37, 53, 10], the map $W(F) \rightarrow W_{n r}(K / F)$ is surjective. Let $\varphi \in I_{n r}^{i+1}(K / F)$ and, by induction on $i$, let $\psi \in I^{i} F$ be such that $\psi_{K}=\varphi$. We have $e^{i}(\psi) \in \operatorname{Ker} \eta_{2}^{i}$, hence $e^{i}(\psi)=c \cdot x$ for some $x \in H^{i-2} F$ by a). By the surjectivity of $e^{i-2}$ and the injectivity of $\bar{e}^{i}$, we can write $\psi=\tau \otimes \theta \perp \psi^{\prime}$, where $\tau$ is the quaternion form corresponding to $c$, $e^{i-2}(\theta)=x$ and $\psi^{\prime} \in I^{i+1} F$. Then $\psi_{K}^{\prime}=\varphi$, as desired.

Notation. We write $\bar{I}^{i} F=I^{i} F / I^{i+1} F$ and, for a finitely generated extension $L / F$, $\bar{I}_{n r}^{i}(L / F)=I_{n r}^{i}(L / F) / I_{n r}^{i+1}(L / F)$.

Lemma C.3. Let $X$ be a quadric (of dimension $>0$ ), and let $C$ be a conic lying on $X$. Let $\tau$ be the quaternion form associated to $C$. Then, for $i \leq 4$, there is a surjection

$$
\tau \bar{I}^{i-2} F(X) \cap \bar{I}_{n r}^{i}(F(X) / F) \longrightarrow \text { Coker } \chi^{i}
$$

Proof. Let $K=F(C)$. Consider the commutative diagram

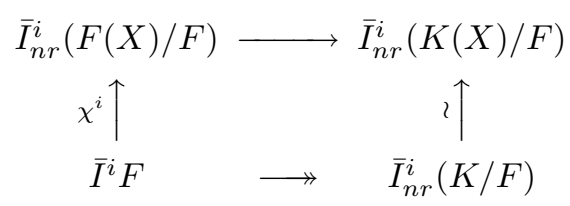

In this diagram, the lower horizontal map is surjective by lemma C.2 b) and the right vertical one is an isomorphism because $K(X) / K$ is purely transcendental. A diagram chase then yields a surjection

$$
\operatorname{Ker}\left(\bar{I}_{n r}^{i}(F(X) / F) \rightarrow \bar{I}_{n r}^{i}(K(X) / F)\right) \rightarrow \operatorname{Coker} \chi^{i} .
$$

To conclude the proof, it suffices to observe that $\operatorname{Ker}\left(\bar{I}^{i} F(X) \rightarrow \bar{I}^{i} K(X)\right)=$ $\tau \bar{I}^{i-2} F(X)$ by lemma C.2 a) and the bijectivity of $\bar{e}^{i-2}$ and $\bar{e}^{i}$.

Proof of theorem C.1. Consider the commutative diagram

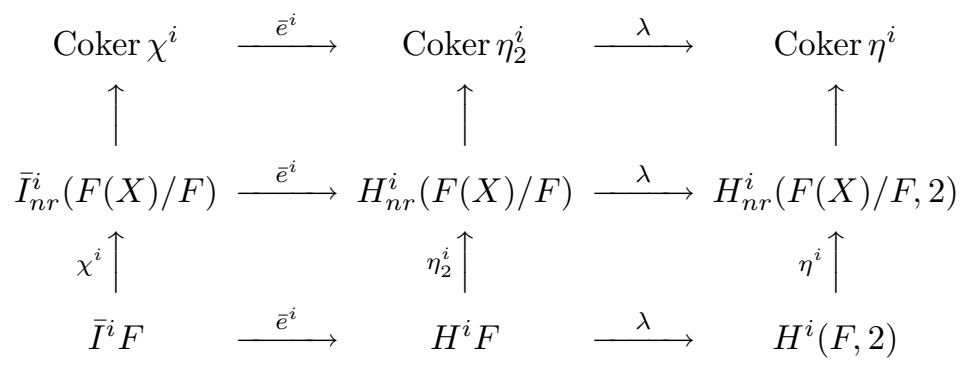

where $\lambda$ is the natural map. Let $x \in \bar{I}_{n r}^{i}(F(X) / F)$. Choose $C$ as in lemma C.3. By this lemma, we can modify $x$ by an element from $I^{i} F$ so that $x=\tau \otimes \theta$. Let 
$y \in H^{i}(F, 2)$ be such that $\lambda \bar{e}^{i}(x)=\eta^{i}(y)$. Let $E / F$ be a quadratic extension such that $C_{E}$, hence $X_{E}$, is isotropic. Then

$$
\tau_{E} \sim 0
$$

hence

$$
x_{E(X)}=0
$$

and

$$
\eta^{i}(y)_{E(X)}=\eta^{i}\left(y_{E}\right)=0 .
$$

Since $X_{E}$ is isotropic, $E(X) / E$ is purely transcendental and $y_{E}=0$ (prop. 2.5 a)). Hence $2 y=0 \in H^{i}(F, 2)$ by the usual transfer argument, and $y=\lambda(z)$ for some $z \in H^{i} F$. By the surjectivity of $\bar{e}^{i}$, write $z=\bar{e}^{i}(t)$ for $t \in \bar{I}^{i} F$. Then

$$
\lambda \bar{e}^{i}(x)=\eta^{i} \lambda \bar{e}^{i}(t)=\lambda \bar{e}^{i}\left(\chi^{i}(t)\right)
$$

hence $x=\chi^{i}(t)$ by the injectivity of $\bar{e}^{i}$ and $\lambda$.

\section{References}

[1] J.Kr. Arason, Cohomologische Invarianten quadratischer Formen, J. Alg. 36 (1975), 448-491.

[2] S.A. Amitsur, Generic splitting fields and central simple algebras, Ann. of Math. 62 (1955), $8-43$.

[3] F.A. Bogomolov, The Brauer group of quotient spaces by linear group actions, Izv. Akad. Nauk. SSSR 51 (1987), 485-516. English translation: Math. USSR Izv. 30 (1988), 455-485.

[4] S. Bloch and A. Ogus, Gersten's conjecture and the homology of schemes, Ann. Sci. Ec. Norm. 7 (1974), 181-202.

[5] J.-L. Colliot-Thélène, Cycles algébriques de torsion et $K$-théorie algébrique, Arithmetical algebraic geometry (Trento, 1991), E. Ballico, ed., Lect. Notes in Math. 1553, Springer, Berlin-Heidelberg-New York, 1993, 1-49.

[6] J.-L. Colliot-Thélène, On the reciprocity sequence in higher class field theory of function fields, in Algebraic K-theory and algebraic topology (P.G. Goerss and J.F. Jardine, eds), NATO ASI Series, Ser. C 407 (1993), 35-55.

[7] J.-L. Colliot-Thélène, Birational invariants, purity and the Gersten conjecture, in: W. Jacob and A. Rosenberg (ed.), $K$-theory and algebraic geometry: connections with quadratic forms and division algebras, Proceedings of Symposia in Pure Mathematics 58 (I) (1995), 1-64.

[8] J.-L. Colliot-Thélène and M. Ojanguren, Variétés unirationnelles non rationnelles: au-delà de l'exemple d'Artin et Mumford, Invent. Math. 97 (1989), 141-158.

[9] J.-L. Colliot-Thélène and R. Parimala, Real components of algebraic varieties and étale cohomology, Invent. Math. 105 (1991), 221-245.

[10] J.-L. Colliot-Thélène and R. Sujatha, Unramified Witt groups of real anisotropic quadrics, in: W. Jacob and A. Rosenberg (ed.), $K$-theory and algebraic geometry: connections with quadratic forms and division algebras, Proceedings of Symposia in Pure Mathematics 58 (II) (1995), 127-147.

[11] P. Deligne, Quadriques, Groupes de Monodromie en géométrie algébrique (SGA 7), exposé XII, Lect. Notes in Math. 340, Springer, Berlin-Heidelberg-New York (1973), 62-81.

[12] J. Dieudonné, Les extensions quadratiques des corps non commutatifs et leurs applications, Acta Math. 87 (1952), 175-242.

[13] R. Elman and T.Y. Lam, Pfister forms and K-theory of fields, J. Alg. 23 (1972), 181-213.

[14] H. Esnault, B. Kahn, M. Levine, E. Viehweg, The Arason invariant and mod 2 algebraic cycles, to appear in J. Amer. Math. Soc.

[15] R.W. Fitzgerald, Witt kernels of function field extensions, Pacific J. Math. 109 (1983), 89106.

[16] W. Jacob and M. Rost, Degree four cohomological invariants for quadratic forms, Invent. Math. 96 (1989), 551-570.

[17] B. Kahn, Lower $\mathcal{H}$-cohomology of higher-dimensional quadrics, Archiv Math. (Basel) 65 (1995), 244-250.

[18] B. Kahn, On the Scharlau transfer, Rocky Mountain J. Math. 19 (1989), 741-747. 
[19] B. Kahn, R. Sujatha, Unramified cohomology of quadrics, II, in preparation.

[20] N. Karpenko, Algebro-geometric invariants of quadratic forms (in Russian), Algebra-i-Analiz 2 (1990), 141-162, 262. English translation: Leningrad Math. J. 2 (1991), 119-138.

[21] N. Karpenko, Cycles of codimension 3 on a projective quadric (in Russian), Zapiski Nauch. Sem. 191 (1991), 114-123. English translation: Journal of Soviet Mathematics 63 (1993), $678-682$.

[22] N. Karpenko, Chow groups of quadrics and index reduction formula, Nova J. of Algebra and Geometry 3 (1995), 357-379.

[23] K. Kato, A Hasse principle for two-dimensional global fields, J. reine angew. Math. 366 (1986), 142-183.

[24] M. Knebusch Generic splitting of quadratic forms, II, Proc. London Math. Soc. 34 (1977), $1-31$.

[25] M. Knus, A.S. Merkurjev, M. Rost, J.-P. Tignol, The book of involutions, in preparation.

[26] T.Y. Lam, The Algebraic theory of quadratic forms (2nd edition), Benjamin, New York, 1980.

[27] A.S. Merkurjev, $K$-theory of simple algebras, in: W. Jacob and A. Rosenberg (ed.), $K$-theory and algebraic geometry: connections with quadratic forms and division algebras, Proceedings of Symposia in Pure Mathematics 58 (I) (1995), 65-83.

[28] A.S. Merkurjev, $S K_{1}$ for biquaternion algebras, preprint, 1994.

[29] A.S. Merkurjev, The group $H^{1}\left(X, \mathcal{K}_{2}\right)$ for projective homogeneous varieties, Algebra i Analiz. English translation: Leningrad (Saint-Petersburg) Math. J. 7 (1995), 136-164.

[30] A.S. Merkurjev, On the norm residue homomorphism for fields, Mathematics in St. Petersburg, 49-71, Amer. Math. Soc. Transl. Ser. 2, 174, Amer. Math. Soc., Providence, RI, 1996.

[31] A.S. Merkurjev and A.A. Suslin, $\mathcal{K}$-cohomology of Severi-Brauer varieties and norm residue homomorphism (in Russian), Izv. Akad. Nauk SSSR 46 (1982), 1011-1046. English translation: Math USSR Izv. 21 (1983), 307-340.

[32] A.S. Merkurjev and A.A. Suslin, The norm residue homomorphism of degree 3 (in Russian), Izv. Akad. Nauk SSSR 54 (1990), 339-356. English translation: Math. USSR Izv. 36 (1991), 349-368.

[33] A.S. Merkurjev and J.-P. Tignol, Galois cohomology of biquadratic extensions, Comment. Math. Helv. 68 (1993), 138-169.

[34] J.W. Milnor, Algebraic K-theory and quadratic forms, Invent. Math. 9 (1969/70), 318-344.

[35] D. Orlov, A. Vishik, V. Voevodsky, Motivic cohomology of Pfister quadrics, in preparation.

[36] I. Panin, Applications of K-theory in algebraic geometry, 1984, Thesis, LOMI, Leningrad.

[37] R. Parimala, Witt groups of anisotropic conics, elliptic and hyperelliptic curves, J. Number Theory 28 (1988), 69-93.

[38] E. Peyre, Unramified cohomology and rationality problems, Math. Ann. 296 (1993), 247-268.

[39] E. Peyre, Products of Severi-Brauer varieties and Galois cohomology, in: W. Jacob and A. Rosenberg (ed.), $K$-theory and algebraic geometry: connections with quadratic forms and division algebras, Proceedings of Symposia in Pure Mathematics 58 (2) (1995), 369-401.

[40] E. Peyre, Corps de fonctions de variétés homogènes et cohomologie galoisienne, C. R. Acad. Sci. Paris 321 (1995), 891-896.

[41] D. Quillen, Higher algebraic K-theory, I, Lect. Notes in Math. 341 (1973), Springer, Berlin, 83-147.

[42] M. Rost, Chow groups with coefficients, Documenta Math. 1 (1996), 319-393.

[43] D.J. Saltman, Noether's problem over an algebraically closed field, Invent. Math. 77 (1984), $71-84$.

[44] D.J. Saltman, Brauer groups and the center of generic matrices, J. of Alg. 97 (1985), 53-67.

[45] W. Scharlau Quadratic and hermitian forms, Grundl. Math. Wiss. 294, Springer, Berlin, 1985.

[46] C. Scheiderer, Real and étale cohomology, Lect. Notes in Math. 1588, Springer, Berlin, 1994.

[47] J-P. Serre, Sur la dimension cohomologique des groupes profinis, Topology 3 (1965), 413-420.

[48] R. Sujatha, Unramified cohomology and Witt groups of anisotropic Pfister quadrics, Trans. Amer. Math. Soc. 349 (1997), no. 6, 2341-2358.

[49] A. Suslin, $K$-theory and $\mathcal{K}$-cohomology of certain group varieties, Algebraic $K$-theory (A. Suslin, ed.), Adv. Soviet Math. 4, AMS, Providence, 1991, 53-74.

[50] R.G. Swan, Zero cycles on quadric hypersurfaces, Proc. Amer. Math. Soc. 107 (1989), 43-46.

[51] M. Szyjewski, The fifth invariant of quadratic forms (in Russian), Algebra Anal. 2 (1990), 213-234. English translation: Leningrad Math. J. 2 (1991), 179-198. 
[52] J.-P. Tignol, $S K_{1}$ for biquaternion algebras, after M. Rost and A. Merkurjev, secret paper, 1994.

[53] J. van Geel, Applications of the Riemann-Roch theorem for curves to quadratic forms and division algebras, preprint, Université catholique de Louvain, 1991.

[54] V. Voevodsky, The Milnor conjecture, preprint, Max-Planck-Institute for Mathematics, Bonn, 1996, http://www.math.uiuc.edu/K-theory/0170,

[55] B.L. van der Waerden, Gruppen von linearen Transformationen, Ergebnisse Math. Wiss., 4, Springer-Verlag, Berlin, 1935.

[56] E. Witt, Über ein Gegenbeispiel zum Normensatz, Math. Zeit. 39 (1935), 462-467.

Institut de Mathématiques de Jussieu, Equipe Théories Géométriques, Université Paris 7, Case 7012, 75251 Paris Cedex 05, France

E-mail address: kahn@math.jussieu.fr

Fakultät für Mathematik, Universität Regensburg, Universitätsstrasse 31, D-93040 Regensburg, Germany

E-mail address: markus.rost@mathematik.uni-regensburg.de

School of Mathematics, Tata Institute of Fundamental Research, Homi Bhabha ROAD, BOMBAY 400005, INDIA

E-mail address: sujatha@math.tifr.res.in 